Florida International University FIU Digital Commons

6-18-2012

\title{
Sensitivity and Uncertainty Analysis of Expected Loss Costs in Hurricane Models
}

Antonio R. Hudson

Florida International University, ahuds001@fiu.edu

DOI: $10.25148 /$ etd.FI12080603

Follow this and additional works at: https://digitalcommons.fiu.edu/etd

\section{Recommended Citation}

Hudson, Antonio R., "Sensitivity and Uncertainty Analysis of Expected Loss Costs in Hurricane Models" (2012). FIU Electronic Theses and Dissertations. 681.

https://digitalcommons.fiu.edu/etd/681

This work is brought to you for free and open access by the University Graduate School at FIU Digital Commons. It has been accepted for inclusion in FIU Electronic Theses and Dissertations by an authorized administrator of FIU Digital Commons. For more information, please contact dcc@fiu.edu. 
FLORIDA INTERNATIONAL UNIVERSITY

Miami, Florida

\title{
SENSITIVITY AND UNCERTAINTY ANALYSIS OF EXPECTED \\ LOSS COSTS IN HURRICANE MODELS
}

\author{
A thesis submitted in partial fulfillment of the \\ requirements for the degree of \\ MASTER OF SCIENCE \\ in \\ STATISTICS \\ by
}

Antonio Ricardo Hudson

2012 
To: $\quad$ Dean Kenneth G. Furton

College of Arts and Science

This thesis, written by Antonio Ricardo Hudson, and entitled Sensitivity and Uncertainty Analysis of Expected Loss Costs in Hurricane Models, having been approved in respect to style and intellectual content, is referred to you for judgment.

We have read this thesis and recommend that it be approved.

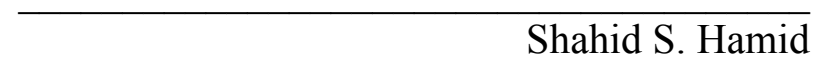

Shahid S. Hamid

B. M. Golam Kibria, Co-Major Professor

Sneh Gulati, Co-Major Professor

Date of Defense: June 18, 2012

The thesis of Antonio Ricardo Hudson is approved.

\begin{tabular}{r}
\hline Dean Kenneth G. Furton \\
College of Arts and Sciences
\end{tabular}

Florida International University, 2012 


\section{ABSTRACT OF THE THESIS \\ SENSITIVITY AND UNCERTAINTY OF EXPECTED LOSS COSTS IN HURRICANE MODELS by}

Antonio Ricardo Hudson

Florida International University, 2012

Miami, Florida

Professor Sneh Gulati, Co-Major Professor

Professor B. M. Golam Kibria, Co-Major Professor

The Public Hurricane Model developed at FIU by a team of scientists has to be certified by the Florida Commission on Hurricane Loss Projection Methodology. The commission ensures that all hurricane loss models meet certain standards, as models are used extensively by regulators and insurance firms to produce inputs used in the homeowner insurance rate making process. The focus of this thesis is to conduct sensitivity and uncertainty analysis through the calculation of standardized regression coefficients and expected percentage reductions in expected loss costs in order to meet the commission standards.

The commission approved the model after very extensive and rigorous review by a panel of experts. The results generated for sensitivity and uncertainty, form S-6, showed the importance of the Holland B parameter regardless of hurricane category, with the radius of maximum winds increasing in importance for stronger hurricanes. 


\section{TABLE OF CONTENTS}

CHAPTER

PAGE

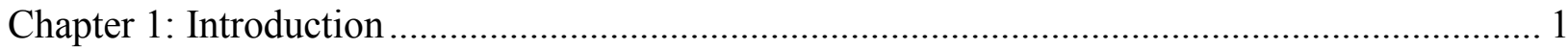

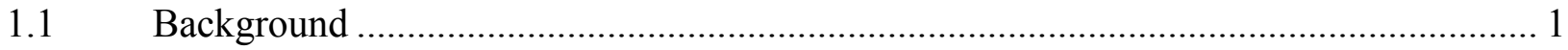

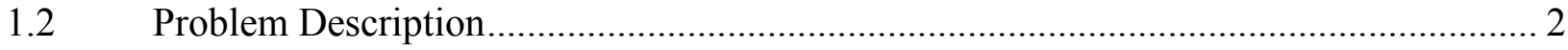

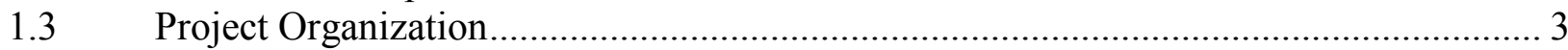

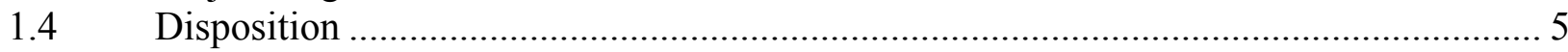

Chapter 2: Statistical Methodology …………………………………………………….....

2.1 Procedure for Calculating Loss Costs form S-5, 2005 ……….................................. 7

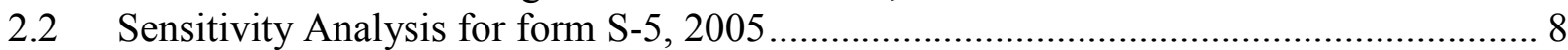

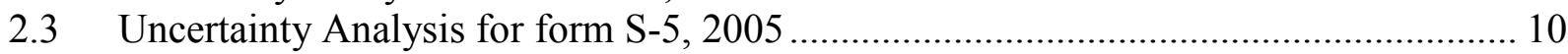

2.4 Sensitivity and Uncertainty Analysis for form S-6, 2010 .......................................... 10

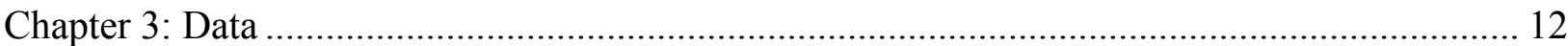

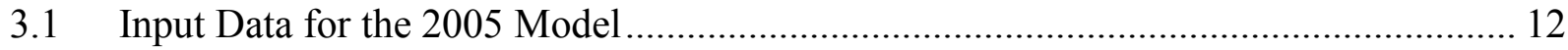

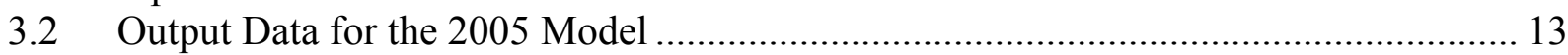

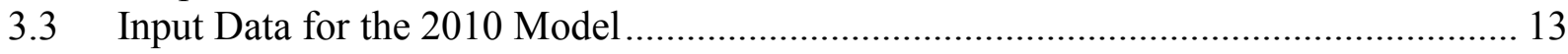

3.4 Output Data for the 2010 Model ............................................................................ 14

Chapter 4: The R Language, Functions Created, and Results ………………………….......... 16

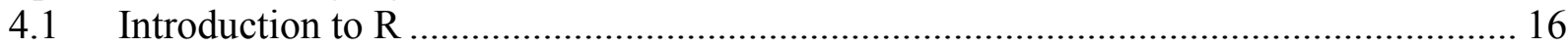

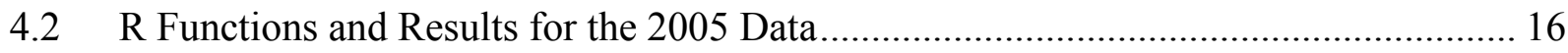

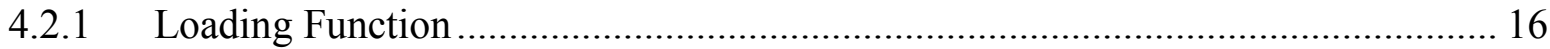

4.2.2 Standardized Regression Coefficients Function and Results............................... 17

4.2.3 Expected Percentage Reduction Function and Results........................................... 18

4.2.4 Contour Plot Function and Results ................................................................ 19

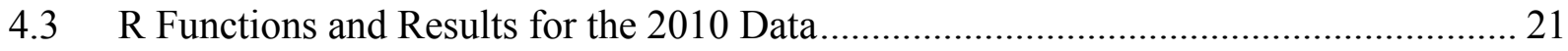

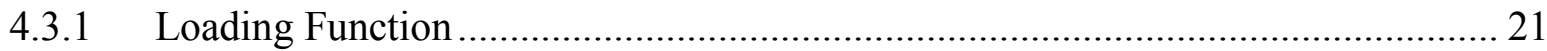

4.3.2 Standardized Regression Coefficients Function and Results................................. 21

4.3.3 Expected Percentage Reduction Function and Results........................................ 22

4.3.4 Contour Plot Function and Results ....................................................................... 23

4.3.5 Cumulative Relative Frequency Function and Results ......................................... 25

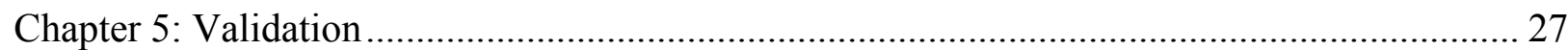

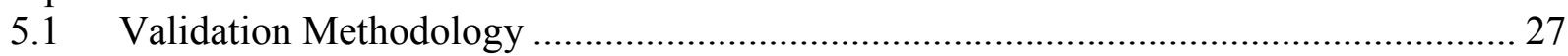

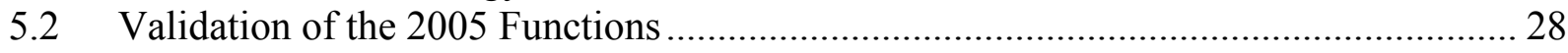

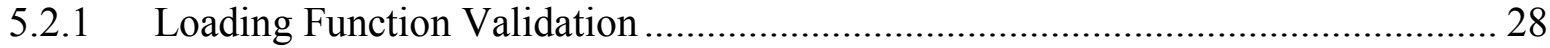

5.2.2 Standardized Regression Coefficients and Expected Percentage Reduction

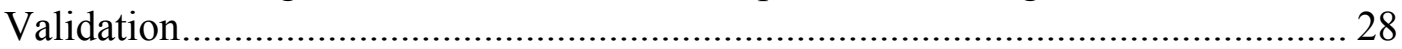

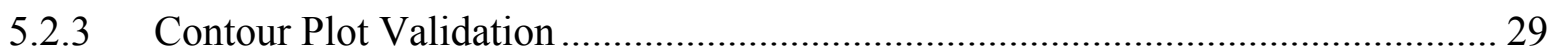

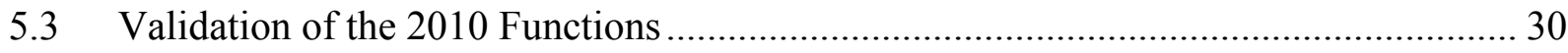

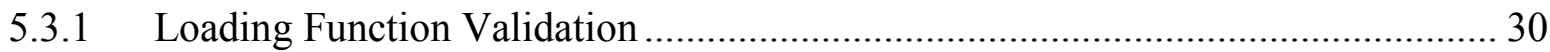




\subsubsection{Standardized Regression Coefficients and Expected Percentage Reduction}

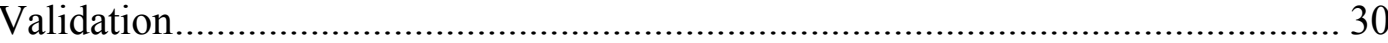

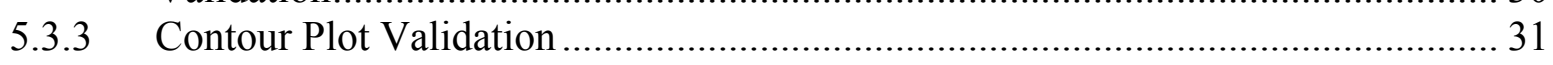

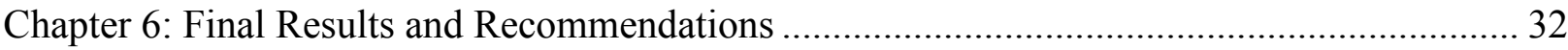

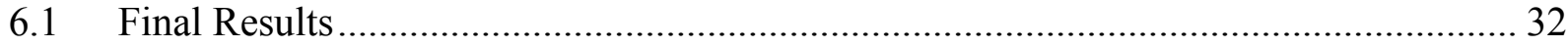

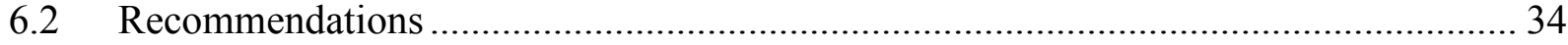

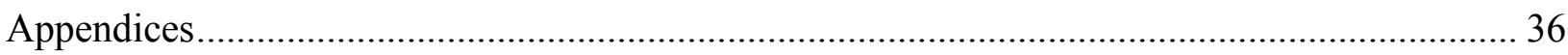

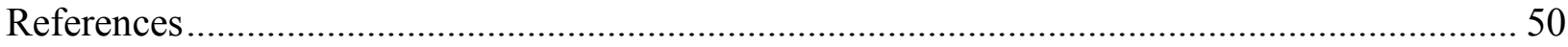




\section{Chapter 1}

\section{Introduction}

\subsection{Background}

The Florida Commission on Hurricane Loss Projection Methodology (FCHLPM) has, as its primary purpose, the regulation of hurricane loss models to ensure that they meet established criteria. The examination of models is required so that "reliable projections of hurricane losses are necessary to assure that rates for residential insurance are neither excessive nor inadequate" (FCHLPM 2007). The commission encourages all models submitted for approval to use computer modeling, and it requires that a series of stringent guidelines be met in order to make sure "that consumers are charged lawful rates for residential property insurance coverage" (FCHLPM 2007).

The Florida Public Hurricane Loss Model (FPHLM) is a joint effort among members of different fields, such as meteorology, engineering, actuarial studies, and statistics, from universities all over the state of Florida. Prior to its conception, the only models available were proprietary models. The programming and design of these models is not publicly available for review, and therefore proper evaluation of the methodology within proprietary models can be difficult. The development of the FPHLM makes it possible to test and compare the results of these proprietary models in order to make sure that insurance costs for the general public are adequately established. Once it had been reviewed and accepted by the commission funding was granted to maintain and update it by the Office of Insurance Regulation (OIR).

The model consists of three separate components: a wind model, a vulnerability model, and an insured loss model. The wind model is dedicated to simulating hurricanes, 
their wind speeds and their decay, once on land, on the basis of historical data. The vulnerability model consists of the engineering simulation of the damage that particular wind speeds inflict upon a structure, its interior, and contents. Finally, the insured loss model is responsible for generating loss costs depending on insurance policy features, and on particular wind speeds and how they affect different construction types. For further details on the FPHLM please refer to Hamid, et al. (2008, 2010).

\subsection{Problem Description}

It is of interest to the analysis of the insured loss model and to the FCHLPM to know the effects that particular predictor variables have on the expected loss cost function. Therefore, it is necessary to conduct sensitivity analysis (SA) and uncertainty analysis (UA) of the loss costs. Sensitivity analysis can be defined as the "study of changes in a model output produced by varying model inputs. This definition includes not only the usual one-at-a-time method, but several classes of techniques" (Los Alamos Scientific Laboratory 1979). Uncertainty analysis is generally defined as the "determination of the variation or imprecision in model output resulting from the collective variation in the model inputs" (Iman et al. 2002a).

The analysis was based on the requirements stipulated by the Florida Commission on Hurricane Loss Projection Methodology. They require the analysis of losses caused by hurricanes of different magnitudes (Category 1, Category 3, and Category 5) to hypothetical $\$ 100,000$ insured structures with zero deductible policies placed approximately three miles apart throughout South Florida. The maximum wind velocity generated in one hour at a point in the South Florida map was calculated using the 
following variables: central pressure, radius of maximum winds, translational velocity, Holland B quantiles, far field pressure, and additional quantiles (these variables are discussed in detail in section 2.1). Maximum wind velocities were then used to calculate the loss cost (LC). Sensitivity analysis and UA were done on the data generated in this fashion (Iman et al. 2002a).

\subsection{Project Organization}

In order to create a set of programming functions capable of doing SA and UA for the 2010 data correctly, it was necessary to create programming functions that could easily be validated and compared to known results. Therefore, the original programming functions created used the 2005 data generated by the FPHLM, for which SA and UA had already been done. Since SA and UA was conducted in 2005 for a previous version of the model and again in 2010 for a new version of the model, the 2005 results were used to thoroughly validate the programming functions before the 2010 model results became available. Furthermore, it also significantly decreased the waiting time between the reception of the 2010 data and the SA and UA results, which could then be used to validate the model and make any appropriate changes. Because of the different format of the 2005 and the 2010 data not all of the functions created for 2005 were used for the 2010 analysis; in some cases it was necessary to modify the 2005 functions in order to address the format changes.

All analysis was done with the R programming language (R Project 2012). Six different functions were created to address the requirements of the FCHLPM: a function capable of loading all necessary files, a function for Sensitivity, a function for 
Uncertainty, a Contour Plotting function, and a Cumulative Relative Frequency function. Each of these functions was created and validated either using the 2005 data in order to mirror the available results from form S-5, Sensitivity and Uncertainty or through traditional programming validation methodology. Form S-5, which lists the requirements for sensitivity and uncertainty according to the FCHLPM in 2005, required sensitivity analysis to be done for LC through the calculation of standardized regression coefficients by hurricane category and each input variable. Uncertainty analysis was carried out through the calculation of expected percentage reductions for LC by hurricane category for each input variable. Contour plots were done for both LC as well as maximum winds for each category hurricane. The Cumulative Relative Frequency distribution compares the hurricane categories by LC (see Chapter 2 for details on form S-5).

Once the 2010 data were received it became apparent that changes to some of the functions were necessary because of changes in the format of the data in 2005 and 2010. Validation of these changes occurred through traditional programming validation methodology as well as through testing the new functions on reformatted 2005 data files made to mimic 2010 data files. After these changes, the files required for form S-6, which specifies the sensitivity by the FCHLPM, were generated. The requirements for form S-6 were very similar to those of form S-5, the differences are discussed in detail in Chapter 2. The results were presented to the evaluating team and form S-6, which was responsible for the SA and UA, was approved after very extensive and rigorous review by a panel of experts.

To summarize, the final project organization was as follows:

1. Receive 2005 data and 2010 requirements (FPHLM form S-6). 
2. Create functions capable of doing all necessary analysis for 2010 requirements using 2005 data.

3. Validation:

a. Validate functions by comparing results to 2005 published results (FPHLM form S-5) where 2005 requirements and 2010 requirements are equivalent.

b. Validate functions through traditional methods where 2010 requirements differ from 2005 requirements so that no 2005 results are available.

4. Receive 2010 data.

5. Modify functions where necessary to deal with data format differences between 2005 and 2010.

6. Validation:

a. Validate functions through traditional methods.

b. Validate functions by testing them on reformatted 2005 data made to mimic 2010 data and comparing to 2005 results.

7. Report SA and UA results.

\subsection{Disposition}

This thesis consists of 6 chapters and 2 appendices. Chapter 1 introduces the problem. Chapter 2 goes through the statistical methodology required to properly analyze the results. Chapter 3 describes in detail the structure, similarities, and differences of the 2005 and the 2010 data. Chapter 4 describes the functions created for forms S-5 and S-6 using the $\mathrm{R}$ statistical programming language. Chapter 5 explains the validation process 
of the functions. Chapter 6 concludes with the final results as well as future recommendations. The appendices contain the fully commented code as well as the complete set of final results presented to the commission. 


\section{Chapter 2}

\section{Statistical Methodology}

\subsection{Procedure for Calculating Loss Costs form S-5, 2005}

The standards specified by the FCHLPM required the FIU team to use a linear model in order to calculate maximum wind velocity per hour $\left(\mathrm{V}_{\max }\right)$. A linear model is of the form:

$$
Y=\boldsymbol{X} \beta+\varepsilon
$$

where the expected value of the response variable, $\mathrm{Y}$, is a linear combination of the vector of parameters, $\beta$. For the hurricane model, the response variable is $V_{\max }$, and the $X$ matrix is populated for a certain category hurricane at a particular vertex point and a certain hour (1 through 12) by the following variables:

$\mathrm{CP}=$ central pressure (in millibars)

$\mathrm{R}_{\max }=$ radius of maximum winds (in statute miles: 1 statute mile $\approx 1.609 \mathrm{~km}$ )

$\mathrm{VT}=$ translational velocity (forward speed in miles/hour)

Holland B Quantiles = model shape parameter

$\mathrm{FFP}=$ far field pressure (in millibars)

Quantiles for possible additional input variables (optional use)

Central pressure refers to the pressure at the center of the hurricane; low central pressure is associated with stronger category hurricanes. $\mathrm{R}_{\max }$ refers to the radius of maximum winds in statute miles; a hurricane with a larger radius has the potential to damage more properties. The translational velocity data gives the forward speed in miles/hour of the hurricane; higher velocities imply a given property spends a shorter amount of time experiencing hurricane force winds. The Holland B parameter is used to better describe the relationship between maximum wind speed and the difference in pressure between 
hurricane center and periphery. It should be noted that modeling the surface pressure field using the Holland B parameter "is a significant improvement over modeling the pressure field with empirical models described by only two parameters" (Vickery \& Wadhera 2008). Far field pressure is the ambient atmospheric pressure outside of the hurricane.

The FCHLPM required the calculation of $\mathrm{V}_{\max }$ given 100 combinations of each of the input variables in order to perform sensitivity and uncertainty analysis. Once $V_{\max }$ was calculated, it was used to compute the loss cost per hour at each vertex based on the \%damage formula given below.

$$
\% \text { damage }=\frac{V_{\max }-50}{140-50}
$$

The percent damage was then used in combination with the loss cost methodology established by the engineering and actuarial models regarding the property type and deductible. This allowed for the calculation of the expected loss costs. However, since the LC calculated is hourly, it was compiled so that "we are looking at maximum total loss costs for each hour for each combination of the (...) variables" (Iman et al. 2002a)

\subsection{Sensitivity Analysis for form S-5, 2005}

Sensitivity analysis was achieved through the study of two statistics that were considered for the linear model. These are the measures of "the relative influence on the magnitude of $\mathrm{V}_{\text {Total }}\left(\mathrm{V}_{\max }\right)$ : partial correlation coefficients (PCC) and standardized regression coefficients (SRC)" (Iman et al. 2002a). Standardized regression coefficients measure how changes in the standard deviation of the independent variables affect the standard deviation of the dependent variable, $\mathrm{V}_{\max }$. A large SRC for a particular independent 
variable implies that $\mathrm{V}_{\max }$ is highly sensitive to changes in the variation of that variable. The calculation of the PCC, which is necessary to calculate the SRC, was done by calculating the correlation of the matrix which includes the 100 values of all input variables as well as the 100 average LC values for each category of hurricane. The correlation matrix R created by the set of correlations can be used to calculate the PCC as follows:

$$
P C C_{X_{i} Y_{j}}=\frac{-R_{X_{i} Y_{j}}^{-1}}{\sqrt{R_{X_{i} X_{i}}^{-1} R_{Y_{j} Y_{j}}^{-1}}}
$$

where $X_{i}$ refers to each one of the above mentioned predictor variables, and $Y_{j}$ refers to each one of the 100 LC values created by the possible combinations for each hurricane. The SRC will be calculated by "multiplying the reciprocal of the element in the lower right hand corner of the matrix $\mathrm{R}^{-1}$ times the first four elements in the last row" (Iman et al. 2002a) of the $\mathrm{R}^{-1}$ matrix.

The SA also required that contour plots be drawn for all models, showing the behavior of different category hurricanes at different locations. In order for plotting to be possible, expected loss costs were averaged over each of the designated vertex points. These points are positioned 3 miles apart in the N-S and E-W directions with $(0,0)$ representing hurricane landfall, creating a map of South Florida. Plotting the distribution of total LC for each hurricane type further allowed us to properly understand how each of the variables affects LC. Finally, the plotting of the SRC at specific times and grid coordinates, although not required for the 2010 submission of the S- 6 form, was used as a validation technique since it was included in the 2005 submission of the S-5 form. 


\subsection{Uncertainty Analysis for form S-5, 2005}

Uncertainty Analysis is centered on the expected percentage reduction (EPR) in the variability of LC created by each one of the predictor variables. Expected percentage reduction values establish much of the variation in the dependent variable, $\mathrm{V}_{\max }$, is caused by each of the independent variables when all other independent variables are held constant. The EPR values show the importance of each of the independent variables in the uncertainty of the dependent variable. The variability reduction is based on the following equation:

$$
\text { Expected \% Reduction }(\mathrm{EPR}) \text { in } \operatorname{Var}(\mathrm{Y})=\frac{\operatorname{Var}\left(E\left(Y \mid X_{j}\right)\right)}{\operatorname{Var}(Y)} \times 100
$$

where $\operatorname{Var}\left(\mathrm{E}\left(\mathrm{Y} \mid \mathrm{X}_{\mathrm{j}}\right)\right)$ is the variance of the expected value of $\mathrm{Y}$ (in this case LC) given only one of the predictor variables, $\mathrm{X}_{\mathrm{i}}$, is present. In order to do this, all other values of the predictor variables in the matrix are substituted by their means. The substitution of each of the predictor variables by their mean allows them to be ranked in order of their expected \% reduction in variability (Iman et al. 2002b). Also, similar to the SA, the plotting of EPR at specific times and grid coordinates, although not required for the 2010 submission, was used as a validation technique.

\subsection{Sensitivity and Uncertainty Analysis for form S-6, 2010}

The SA and UA for form S-6 follow the same statistical methodology as the 2005 procedure. Changes include that the number of vertices was reduced for form S-6 as well as the fact that different input variables were used and LC were calculated directly. These changes in the requirements made by the FCHLPM meant that SA and UA were done on 
LC only. As required by the commission, the final report showed the UA and SA through the calculations of EPR, SRC, cumulative relative frequency (CRF) for Averaged Expected LC. The commission also required contour plots for all of the predictor variables in order to properly establish the relevance of each one in terms of sensitivity and uncertainty when estimating LC for each hurricane category. 


\section{Chapter 3}

\section{Data}

\subsection{Input Data for the 2005 Model}

The data the model used in 2005 to generate maximum wind velocities and expected loss costs consist of an input file for each category Hurricane (one, three, and five) which contains 100 values for the following input variables: CP, Rmax, VT, Quantile 1 (Holland B), and Quantile 2 (a description of the variables can be found in the statistical methodology section). It should be noted that the Holland B quantiles are not Holland B values, which were not readily available for the 2005 data. Additional input files were generated for each variable, which were used in order to calculate the expected percentage reduction (EPR) values for UA. In each of these files all other variables were replaced with the mean of the 100 values for that variable, allowing for the analysis of the effects of a single variable on LC. In total there were six input files (For inspection, these files can be seen in appendix 2: Data Files).

Table 1: Sample of 2005 input file, all-variable

\begin{tabular}{|r|r|r|r|r|}
\hline \multicolumn{1}{|c|}{ CP } & \multicolumn{1}{|c|}{ Rmax } & VT & Quantile 1 & Quantile 2 \\
\hline 979.5 & 28.76 & 12.97 & 0.6762 & 0.9657 \\
\hline 982.4 & 31.35 & 16.45 & 0.0041 & 0.7213 \\
\hline 988.8 & 22.05 & 17.59 & 0.0663 & 0.6394 \\
\hline 979.4 & 26.44 & 16.99 & 0.9609 & 0.6695 \\
\hline
\end{tabular}

Table 2: Sample of 2005 additional input file for EPR, only CP variable

\begin{tabular}{|r|r|r|r|r|}
\hline \multicolumn{1}{|c|}{ CP } & \multicolumn{1}{|c|}{ Rmax } & VT & Quantile 1 & Quantile 2 \\
\hline 979.5 & 24.66 & 15.00 & 0.5006 & 0.4999 \\
\hline 982.4 & 24.66 & 15.00 & 0.5006 & 0.4999 \\
\hline 988.8 & 24.66 & 15.00 & 0.5006 & 0.4999 \\
\hline 979.4 & 24.66 & 15.00 & 0.5006 & 0.4999 \\
\hline
\end{tabular}




\subsection{Output Data for the 2005 Model}

The output data consist of one output file for each category hurricane and input file type except Quantile 2, which was not used in the model, so that in total each category hurricane has six input and five output files. Each output file contains wind velocities, where each row specifies a particular vertex point on the map; the vertex points are distributed in three mile intervals from 0 to $135 \mathrm{E}-\mathrm{W}$ and -15 to $45 \mathrm{~S}-\mathrm{N}$, where $(0,0)$ represents hurricane landfall in Miami, Florida. Each column of the output file specifies a particular hour of the day, with the final column being the average wind velocity at that particular vertex point. There are 966 vertex points and 100 values per point, such that each column has a total of 966,000 wind velocities. Also of paramount importance is a separate file which labels whether the vertex point is in land or water (For inspection, these files can be seen in appendix 2: Data Files).

Table 3: Sample of 2005 output file used

\begin{tabular}{|r|r|r|r|r|r|r|r|r|r|r|r|r|r|r|r|r|}
\hline Input & E-W & S-N & H 0 & H 1 & H 2 & H 3 & H 4 & H 5 & H 6 & H 7 & H 8 & H 9 & H 10 & H 11 & H 12 & Max \\
\hline 1 & 0 & -15 & 51.7 & 64.1 & 72.9 & 67.5 & 56.8 & 48 & 41.1 & 35.6 & 31.5 & 26.8 & 24.7 & 23 & 0 & 72.9 \\
\hline 1 & 0 & -12 & 44.2 & 57.6 & 75.4 & 69.1 & 57.9 & 48.8 & 41.7 & 36.1 & 31.9 & 27.1 & 24.9 & 23.3 & 0 & 75.4 \\
\hline 1 & 0 & -9 & 35.4 & 52.5 & 77.3 & 70.5 & 58.9 & 49.6 & 42.3 & 36.6 & 32.2 & 27.4 & 25.2 & 23.5 & 0 & 77.3 \\
\hline 1 & 0 & -6 & 24.8 & 49.1 & 78.8 & 71.6 & 59.8 & 50.3 & 42.8 & 37 & 32.6 & 27.6 & 25.4 & 23.7 & 0 & 78.8 \\
\hline
\end{tabular}

\subsection{Input Data for the 2010 Model}

The 2010 input data for form S-6 is identical to the 2005 data for form S-5, with the exception that the input variables are different. In 2010 the variables used were $C P, R_{\max }$, VT, FFP, and Holland B (a description of the variables can be found in the statistical methodology section). Similarly to the 2005 input files, additional input files were generated for EPR calculations, so that there are a grand total of six input files. 
Additionally, a separate file was given which makes it possible to find the exact values for the Holland B parameter given the quantiles in the input file (For inspection, these files can be seen in appendix 2: Data Files).

Table 4: Sample of 2010 input file, all-variable

\begin{tabular}{|r|r|r|r|r|}
\hline \multicolumn{1}{|c|}{ CP } & $\mathbf{R}_{\max }$ & VT & Holland B Q & \multicolumn{1}{c|}{ FFP } \\
\hline 984.5 & 26.56 & 18.43 & 0.6599 & 1011.27 \\
\hline 982.2 & 25.72 & 14.19 & 0.3269 & 1017.08 \\
\hline 987.5 & 28.76 & 12.00 & 0.4751 & 1018.13 \\
\hline 978.4 & 30.82 & 17.96 & 0.0794 & 1007.43 \\
\hline
\end{tabular}

Table 5: Sample of 2010 additional input file for EPR, only $R_{\max }$ variable

\begin{tabular}{|r|r|r|r|r|}
\hline \multicolumn{1}{|c|}{ CP } & $\mathbf{R}_{\max }$ & VT & Holland B Q & \multicolumn{1}{c|}{ FFP } \\
\hline 982.5 & 26.56 & 15.00 & 0.5006 & 1013.00 \\
\hline 982.5 & 25.72 & 15.00 & 0.5006 & 1013.00 \\
\hline 982.5 & 28.76 & 15.00 & 0.5006 & 1013.00 \\
\hline 982.5 & 30.82 & 15.00 & 0.5006 & 1013.00 \\
\hline
\end{tabular}

\subsection{Output Data for the 2010 Model}

The output files produced were markedly different from the 2005 results. Instead of having separate files for each hurricane, all hurricane categories were given within a single file; also, the files no longer report wind velocities, but instead report LC directly. These LC values were aggregated throughout time and averaged in two separate ways, creating two output files per input file. The first method averaged LC over all map vertex points, such that $100 \mathrm{LC}$ values were given as output; the second method averaged LC over the 100 values for each vertex point while removing all water points, so that $682 \mathrm{LC}$ values were given as output, one for each point on the map. The reason for this is that the 
map was shortened from 2005 to 2010 so that it is now from 0 to $117 \mathrm{E}-\mathrm{W}$ and -15 to 45

S-N (For inspection, these files can be seen in appendix 2: Data Files).

Table 6: Sample of 2010 output file by input

\begin{tabular}{|r|r|r|r|}
\hline \multicolumn{1}{|c|}{ Cat } & \multicolumn{1}{|c|}{ Input } & ID & LC \\
\hline 1 & 1 & 2345036 & 3.44 \\
\hline 1 & 2 & 2772487 & 4.07 \\
\hline 1 & 3 & 2228470 & 3.27 \\
\hline
\end{tabular}

Table 7: Sample of 2010 output file by coordinates

\begin{tabular}{|r|r|r|c|}
\hline \multicolumn{1}{|c|}{ Cat } & \multicolumn{1}{|c|}{ E-W } & \multicolumn{1}{c|}{ S-N } & \multicolumn{1}{c|}{ LC } \\
\hline 1 & 6 & 33 & 8.3585 \\
\hline 1 & 6 & 36 & 6.9207 \\
\hline 1 & 6 & 39 & 5.6162 \\
\hline
\end{tabular}




\section{Chapter 4}

\section{The R Language, Functions Created, and Results}

\subsection{Introduction to $\mathbf{R}$}

" $\mathrm{R}$ is a system for statistical computation and graphics. It consists of a language plus a run-time environment with graphics, a debugger, access to certain system functions, and the ability to run programs stored in script files" (R Project 2012). Due to its nature, R contains a very large library of statistical functions. It also allows the user to create functions to analyze large data sets. The open source nature of $\mathrm{R}$ makes it a trustworthy software for calculations, since any sufficiently competent observer can enquire fully about what is actually being computed. "There are no intrinsic limitations to the validation of the software, in the sense that it is all there" (Chambers 2008). Furthermore, “open-source systems demonstrably generate a spirit of community among contributors and active users (...) with a great deal of effort devoted to testing and extension of the systems" (Chambers 2008). Therefore, the large variety of packages readily available for

download and the thorough validation of both $\mathrm{R}$ and all relevant packages make $\mathrm{R}$ a perfect tool to fulfill the S-6 requirements of the FCHLPM for sensitivity and uncertainty analysis.

\subsection{R Functions and Results for the 2005 Data}

\subsubsection{Loading Function}

In order to perform both SA and UA, several different files had to be loaded into the $\mathrm{R}$ interface before any analysis could take place. Therefore, the first piece of code written, the loading loop, is one that allows for both the loading of all necessary files and, at the same time, differentiates between input and output files while numbering them. 
The differentiation is possible because all input files are .csv files, while all output files are .dat files. Since the loading loop is searching for all files within a particular folder, it follows the same order as the order of the files in the folder. Therefore the first file in the folder, assuming it is an input file, would be input 1 within the $\mathrm{R}$ console. The loading and sorting allows for clear distinction between files and they are now ready to be analyzed.

\subsubsection{Standardized Regression Coefficients Function and Results}

The SRC function as created with the data used for the S-5 form was designed so that a series of matrices could be analyzed, one for each hurricane, and combined into a single matrix of results. For the S-5 form, each matrix was $100 \times 5$, and the final SRC matrix was $5 \times 3$. This required the attaching of 100 wind velocity values to the end of the original input file as well as the removal of Quantile 2 (See Figure 1). In order to do this, 966,000 wind velocities had to be sorted and averaged. For each input value, the average over all land based vertex points was found, so that each wind velocity value used is the average over time and over all land based vertex points. Once this column is available, the statistical methodology details how the inverse of the correlation matrix is calculated and then the ratio of the correlations give the SRC values in matrix format. The repetition of this procedure for each hurricane category meant that the matrix produced had 5 rows, one for each variable, and three columns, one for each hurricane category.

An alternate version of the SRC function was also created that is capable of calculating the SRC as described above but at a particular vertex point. Vertex point SRC calculation was required for form S-5 but not S-6, so it was done in order to validate the code. Further discussion of this function can be found within the validation chapter, since 
it was one of the results available for the S-5 form. The final results required a graphical representation of the SRC matrix, so a graphing function was created capable of turning the matrix into a line plot, as shown below.

Table 8: SRC matrix based on 2005 data

\begin{tabular}{|c|c|c|c|c|}
\hline Category & $C P$ & $R \max$ & $V T$ & $\begin{array}{c}\text { Holland } \\
B\end{array}$ \\
\hline 1 & -0.43 & 0.23 & 0.16 & 0.71 \\
\hline 3 & -0.26 & 0.22 & 0.17 & 0.82 \\
\hline 5 & -0.20 & 0.84 & -0.01 & 0.43 \\
\hline
\end{tabular}

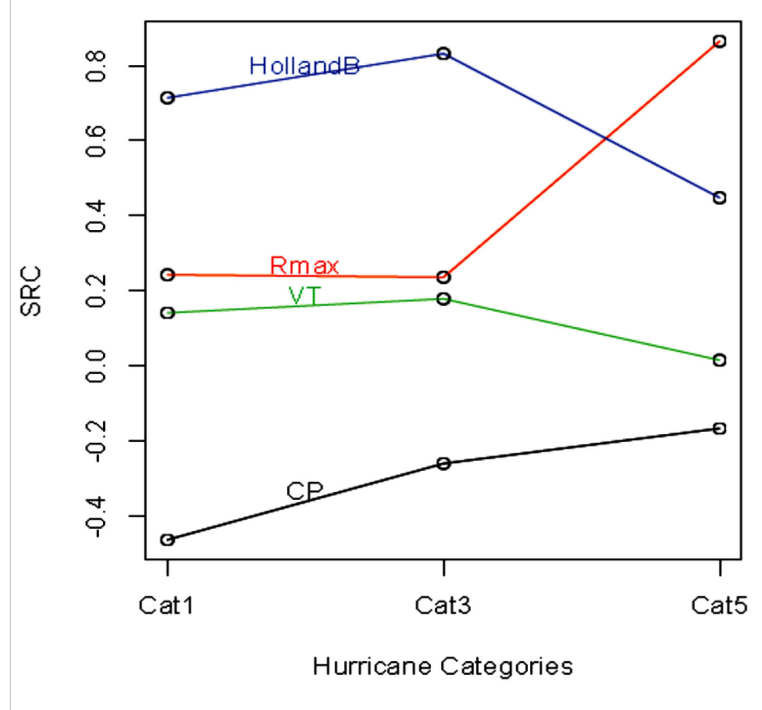

Figure 1: SRC derived from 2005 data

\subsubsection{Expected Percentage Reduction Function and Results}

The EPR function works with the average over all land based vertex points and over time of the wind velocities. It does this for both all-variable output files as well as output files produced when only a single variable remained unchanged while all other variables were constant. Each of the three hurricane categories used require the same calculations. Once said calculations have taken place, as stated in the statistical 
methodology, the variance of each column, where each column represents a different output file, is calculated and then the ratio of each variance in relation to the variance of the all-variable output file is calculated. The output is then stored into a $5 \times 3$ matrix, one row for each variable, one column for each hurricane category.

Much like the alternate SRC function, an alternate EPR function was also created capable of calculating the EPR at a particular vertex point. Further discussion of this function can be found within the validation chapter, since it was one of the results available for the S-5 form. Furthermore, since the final results for EPR also required a graphical representation of the EPR matrix, the same simple graphing function used for SRC was used for EPR in order to turn the matrix into a line plot, as shown below.

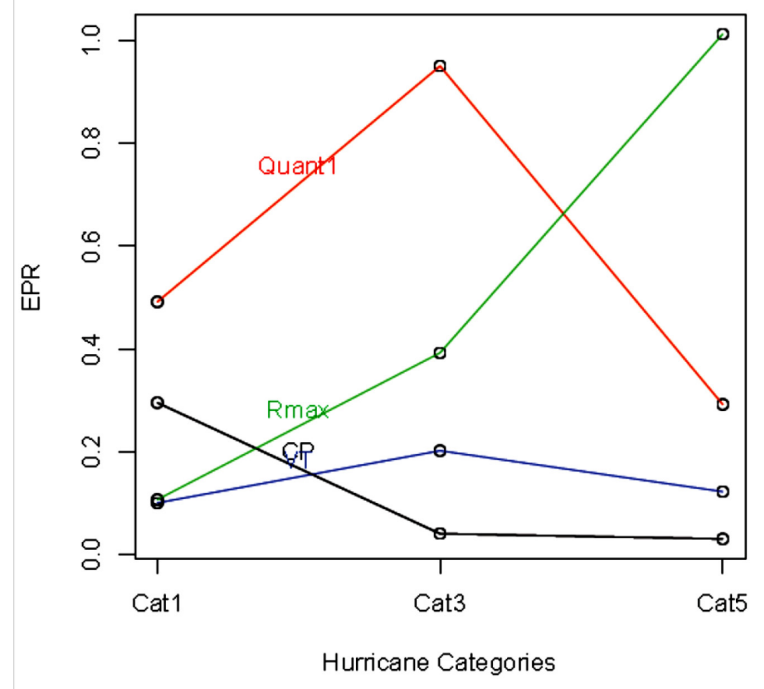

Figure 2: EPR at (30,3) derived from 2005 data

\subsubsection{Contour Plot Function and Results}

The Contour Plot function, when dealing with wind velocities from the output files, treats these velocities by finding the average over all input values and time, so that there is a single value for each vertex point, where non-land vertex points are replaced 
with zeroes. The function then generates a $46 \times 21$ matrix of the 966 vertex points, with E-W as rows and N-S as columns. The order of all the rows is then inverted from E-W to W-E in order to make sure that the R plotting function, which graphs from left to right, plots the wind velocities correctly. The R plotting function used divides the wind speeds into equal intervals and sorts them by color, with deeper shades of red implying higher velocities, so that a full map of wind velocities for a particular category hurricane in South Florida is created.

Table 9: Sample of sorted matrix based on 2005 data used for Contour Plot

\begin{tabular}{|r|r|r|r|r|}
\hline N-S $\backslash \mathbf{E}-W$ Coordinates & $\mathbf{3 9}$ & $\mathbf{4 2}$ & $\mathbf{4 5}$ & $\mathbf{4 8}$ \\
\hline $\mathbf{0}$ & 64.55 & 64.471 & 64.153 & 64.226 \\
\hline $\mathbf{3}$ & 65.893 & 66.889 & 66.725 & 66.792 \\
\hline $\mathbf{6}$ & 67.518 & 68.309 & 68.115 & 68.018 \\
\hline $\mathbf{9}$ & 69.346 & 68.848 & 68.309 & 68.115 \\
\hline
\end{tabular}

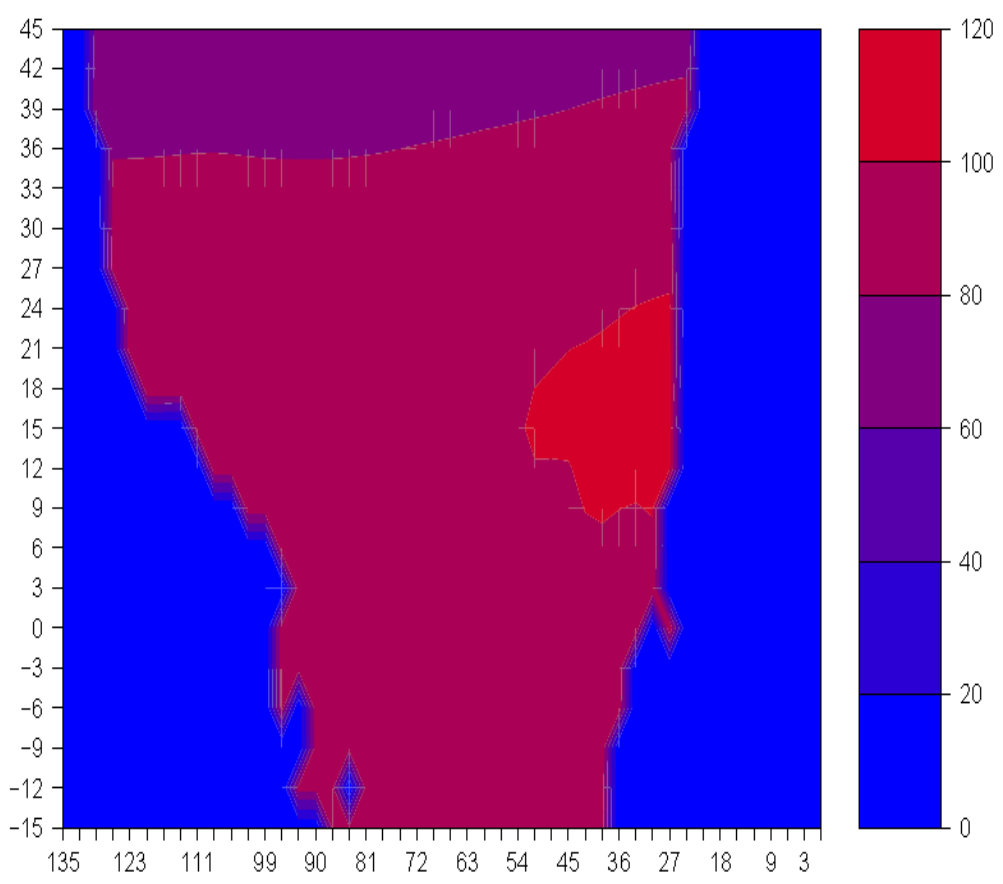

Figure 3: Contour Plot of Maximum Velocity for Category 3 Hurricane 


\subsection{R Functions and Results for the 2010 Data}

\subsubsection{Loading Function}

The loading function used for the 2010 data is almost an exact carbon copy of the 2005 loading function. The only difference is that the output files produced by the 2010 model are not .dat files, but .txt files. Therefore the function now sorts input files versus output files on the basis of whether the file is a .csv file (input) or a .txt file (output).

\subsubsection{Standardized Regression Coefficients Function and Results}

The second version of the SRC function, as created for the S-6 form data, is markedly different from its 2005 counterpart because the output files of the model are so different. The LC values in the output files were already calculated, averaged and returned in sets of 100 for each hurricane category, so that the sorting and averaging of large sets of wind velocities was no longer necessary. Furthermore, since the Holland B values used were made available, it was necessary to replace the Holland B quantile values given in the input file with their actual values, as described in section 3.2. Once

these two differences were taken into account, the formation of the $100 \times 6$ matrix of input files and output results was straightforward. With the $100 \times 6$ matrix the code that calculates the SRC is the same as the 2005 version, which follows the pre-established methodology. The final result is a 5 x 3 matrix for each variable and hurricane category. The final matrix was graphed using the same function that was used for the 2005 results. 


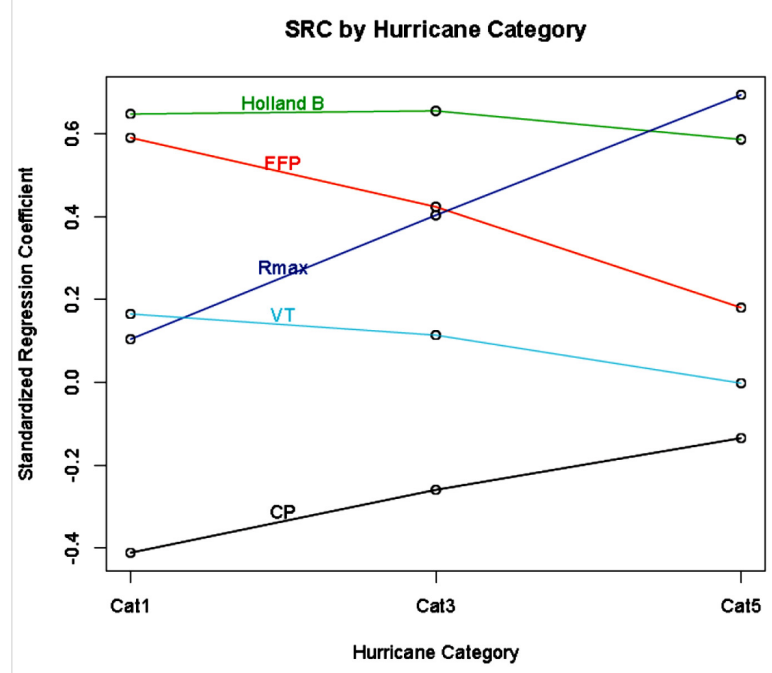

Figure 4: Final Submissions of SRC for form S-6

The final SRC submission shows that, for a category 1 hurricane, changes in the standard deviation of the Holland B parameter and the Far Field Pressure have the largest positive effect on changes on LC deviation. However, as hurricane category increases, Holland B remains significant while FFP decreases significantly. $\mathrm{R}_{\max }$ and VT are not nearly as influential for a category 1 , although $\mathrm{R}_{\max }$ increases significantly in influence on the standard deviation of LC for category 3 and category 5 hurricanes. Central pressure is of particular interest when looking at category 1 hurricane results due to the strong negative relation between $\mathrm{CP}$ and expected loss costs. This relationship decreases as the hurricane increases in intensity.

\subsubsection{Expected Percentage Reduction Function and Results}

Similar to the SRC version for form S-6, the main distinction between the EPR function for form S-6 and the 2005 version is the format of the output files. Since the LC results were given directly in the output files and they were already averaged, the creation of a matrix of LC results for the different output files is almost immediate, at which point 
the EPR calculations are done using the same process as the 2005 version, based on the pre-established methodology. This creates a $5 \times 3$ matrix for each variable and hurricane category. The final matrix was graphed using the same function that was used for the 2005 results.

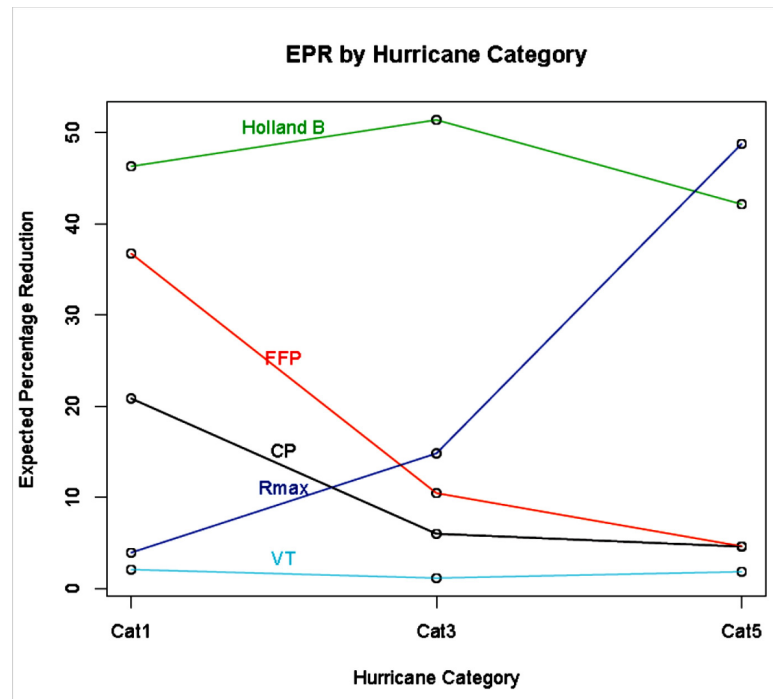

Figure 5: Final Submissions of EPR for form S-6

The final submission for EPR shows that the Holland B parameter and the FFP are largely responsible for the variance of LC for a category 1 hurricane, with $\mathrm{CP}$ being less significant, and $\mathrm{R}_{\max }$ and VT being insignificant. Category 3 hurricanes continue to have the Holland $B$ parameter as predominantly significant, with $R_{\max }$ gaining significance while all other variables lose it. Category 5 hurricane variance of LC is predominantly explained by $\mathrm{R}_{\max }$ and the Holland $\mathrm{B}$ parameter, while all other variables are insignificant.

\subsubsection{Contour Plot Function and Results}

The Contour Plot function that analyzed the data given to perform S-6 is different from the 2005 version since the output file used for the contour plots already has the LC 
calculated and averaged over the 100 input values. The 2010 data contains LC for each vertex point excluding all water based points. In order to properly plot the LC it is therefore necessary to reintroduce the water based points so a complete matrix for E-W and N-S vertex points can be generated. Once this is achieved the plotting mechanism is the same as the one used to plot the 2005 contour plots.

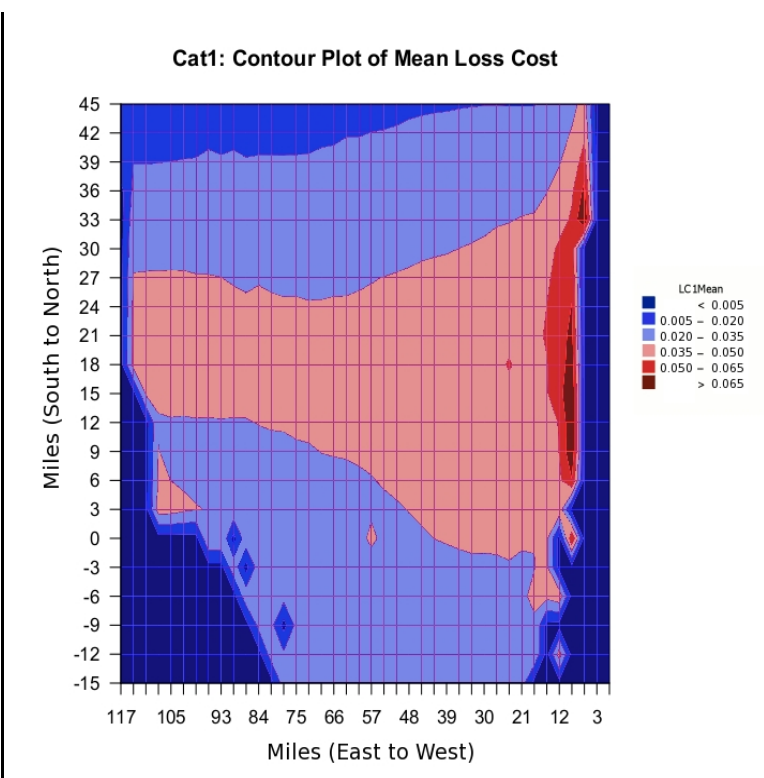

Figure 6: Cat 1 Contour Plot of Mean Loss

Cost based on 2010 data

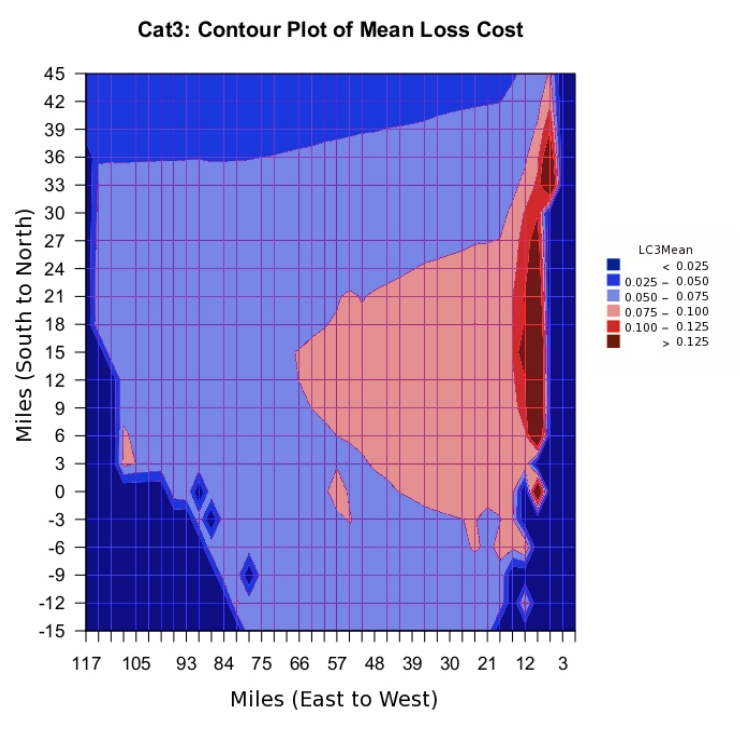

Figure 7: Cat 3 Contour Plot of Mean Loss Cost based on 2010 data 


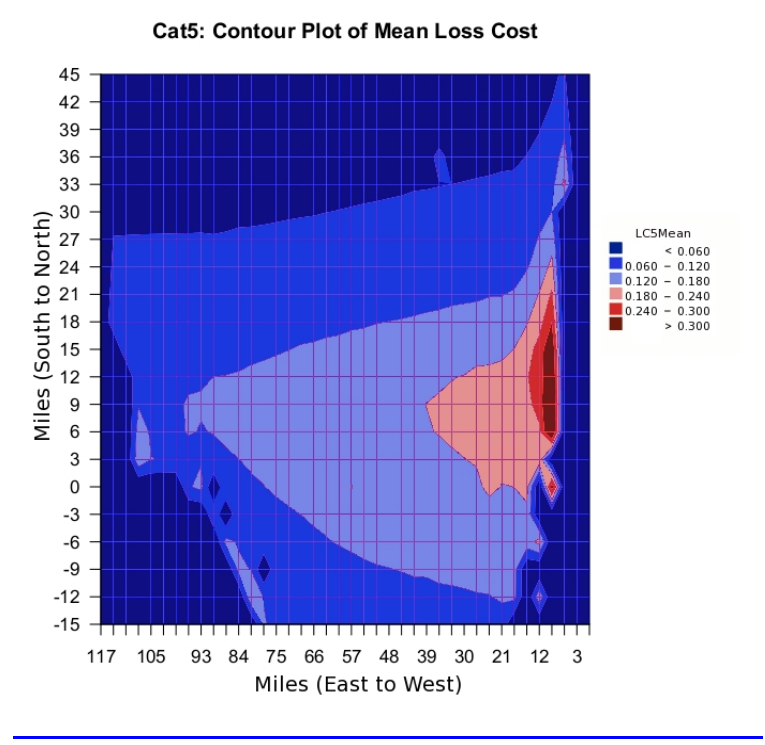

Figure 8: Cat 5 Contour Plot of Mean Loss Cost based on 2010 data

The contour plots show the mean LC across South Florida presented as a proportion of the total exposure. The category 1 hurricane contour plot shows maximum mean LC of .065 , is contained primarily in the immediate landfall area. The category 3 contour plot shows mean LC greater than .05 throughout most of the map, with a maximum mean LC of .120 in the immediate landfall area. The category 5 contour plot shows a mean LC greater than .12 stretching from east to west through the center of South Florida, with a maximum mean LC of .300 in the immediate landfall area. The stark differences in the plots show how an increase in hurricane intensity implies a significant increase in mean expected loss costs.

\subsubsection{Cumulative Relative Frequency Function and Results}

The Cumulative Relative Frequency function used is a preexisting $\mathrm{R}$ function that was formatted to fit the necessary plotting needs of form S-6. No earlier version was created when working with the data from form S-5 due to the straightforward nature of 
the task. Validation was done through functional testing. Predetermined data sets of data were created to compare function results with expected results; all testing proved successful.

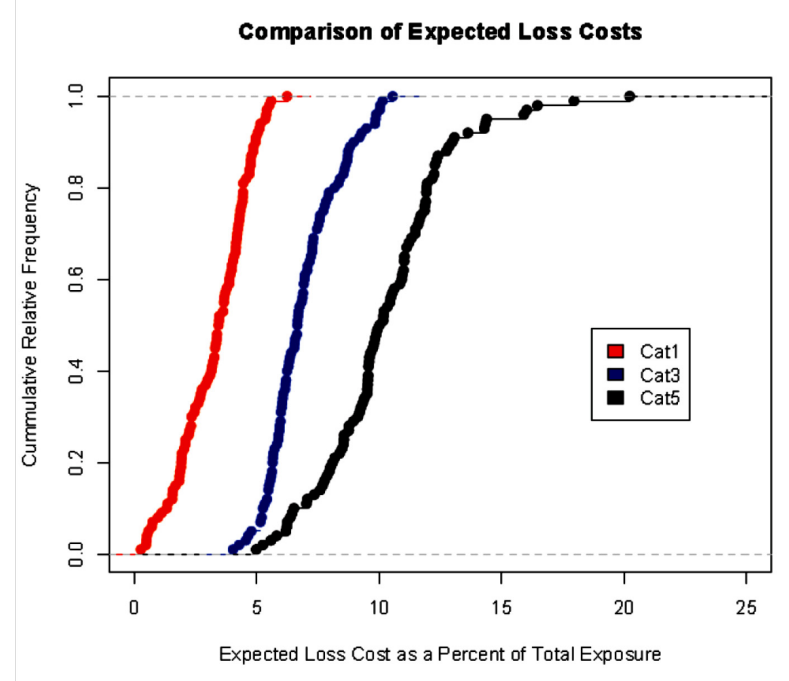

Figure 9: CRF based on 2010 data

The cumulative relative frequency graph produced for the final submission shows that, as a percentage of total exposure, expected loss costs for a category 5 are significantly larger than those of a category 1 or a category 3 hurricane. 


\section{Chapter 5}

\section{Validation}

\subsection{Validation Methodology}

Whenever computer programming methods are used to calculate results, the importance of validating the code cannot be understated. Without proper validation it is impossible to be certain of the results generated by any of the functions written. Each piece of the code must therefore be validated separately, and even functions that are part of $\mathrm{R}$ libraries were checked to make sure all results were valid despite previous validation by the $\mathrm{R}$ community.

The two methods used to validate the $\mathrm{R}$ functions created were either through direct comparison to results from form S-5, or through traditional validation methods such as formal testing, functional testing or random testing (Tran 1999). The main method used when results from form S-5 were not available due to the differences between requirements for forms S-5 and S-6 was a walk-through. Walk-throughs consist of running through a function one line of code at a time, in order to see if the expected results are generated in a piece-meal fashion. The second method used, aside from the walk-through, was the testing of functions with test-case files in order to see if predetermined results were generated. 


\subsection{Validation of the 2005 Functions}

\subsubsection{Loading Function Validation}

The loading loop was validated through a simple process. Any of the files that were loaded into R were also opened through Windows Excel. Making sure that the files were properly loaded was merely a matter of matching the files in $\mathrm{R}$ with the Windows Excel files. Since the function being used to read each of the files was part of the main $\mathrm{R}$ library, its validation was not required. It was, however, necessary to check that all input and output files had been loaded and correctly differentiated in order to make sure that, when other functions called on them, the correct files would be brought forth.

\subsubsection{Standardized Regression Coefficients and Expected Percentage Reduction} Validation

Validating the SRC and the EPR code for form S-5 was done in several stages. First, it was necessary to make sure that the sorting and averaging of all vertex points so that a set of 100 average wind velocities could be used was done correctly. Because $\mathrm{R}$ is a functional language, this was validated by running the code one line at a time and then randomly comparing some of the sorted sets of values with the original unsorted ones. Once walk-through validation was done, and knowing that functions like mean or variance are inherent to $\mathrm{R}$, final validation of the $\mathrm{SRC}$ and the EPR code was done by comparing the wind velocity results produced by the functions with the original 2005 S-5 SRC and EPR results based on expected loss costs. Because of the close relationship between wind velocity and LC, it was expected that the two graphs would be extremely similar. 
The SRC and the EPR were further validated through the creation of variations on the code to allow for the analysis of wind velocities both at particular vertex points and throughout time intervals. Both of these were also compared to S-5 SRC and EPR results based on the 2005 expected loss costs. Further validation was done through the creation of test-case files where all variables were made constant to see if the SRC and EPR calculated returned the expected results.

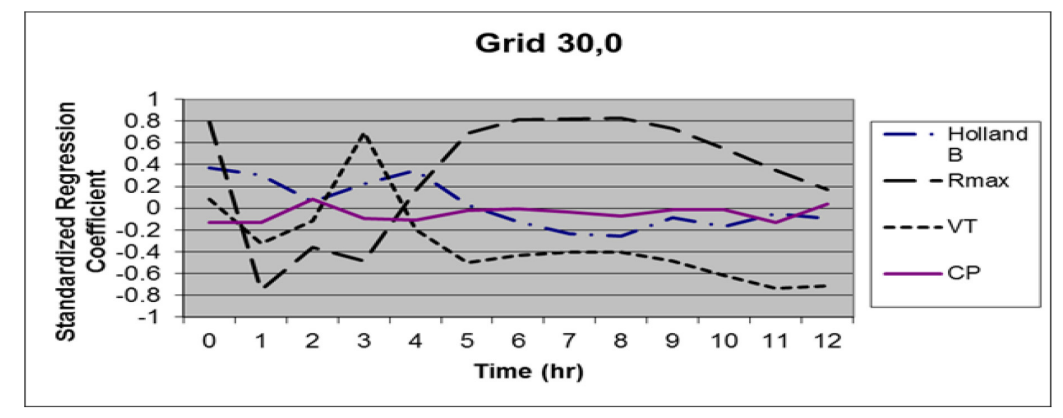

Figure 10: SRC for LC at (30,0) as submitted for form S-5in 2005

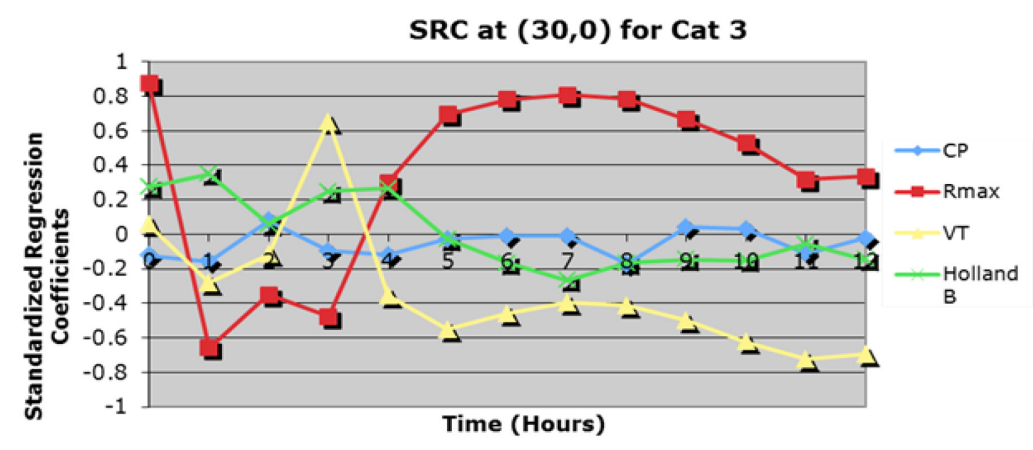

Figure 11: SRC for $V_{\max }$ at (30,0) as generated for validation based on the data for form S-5in 2005

\subsubsection{Contour Plot Validation}

Validating the S-5 Contour Plot function was extremely similar to the validation of the SRC and EPR functions since validation of averages calculated had to take place. For the Contour Plot function, averages were calculated over all input values to return a mean $\mathrm{V}_{\max }$ for each vertex point. Randomized testing of the averages generated for some 
of the 966 vertices showed consistency. After this, the replacement of average $V_{\max }$ values with zeroes where water was present according to the Land-Water ID file was done and then the contour plot function used to graph was a part of a pre-existing $\mathrm{R}$ package. The ultimate validation of the S-5 Contour Plot function is the Contour Plot itself, which is a clear representation of south Florida and can be easily read and compared to the 966 average vertex values.

\subsection{Validation of the 2010 Functions}

\subsubsection{Loading Function Validation}

Since the only change required in the loading function was a single line of code that specified that output files from the model were .dat, which was changed to .txt for the 2010 results, no validation other than the comparison between loaded files and the actual files was deemed necessary.

\subsubsection{Standardized Regression Coefficients and Expected Percentage Reduction Validation}

The 2010 versions of the SRC and EPR functions required the removal of the initial part of the code that sorted and averaged out the data, since this was already done to the output files. Other than that the functions remained unchanged, and therefore

previous validation was deemed appropriate. However, due to the importance of having results that were correct, a walk-through of the code was once again done to corroborate the results. 


\subsubsection{Contour Plot Validation}

The validation of the S-6 Contour Plot function was done particularly carefully, since results generated by the original S-6 output files did not reflect what was expected by the modeling team; it was later discovered that there were inaccuracies in the output files generated by the model and new files were generated. The major changes in the output files made the validation very straightforward since the output file used for form S-6 was already averaged for each vertex, making it unnecessary for the function to do so. The removal of water vertices in their entirety in the output file made it necessary to reintroduce zeroes representing water vertices in order to properly plot the south Florida area. Validation of the S-6 Contour Plot was done by comparing it to the S-5 Contour Plot to show that both plots depict a map of South Florida when using a test file with 0 for water values and 1 for land values. The only distinction is the removal of a large

portion of the water vertex values that are east of Florida for form S-6 when compared to form S-5. 


\section{Chapter 6}

\section{Final Results and Recommendations}

\subsection{Final Results}

The focus of the previously described analysis was the necessity of analyzing the linear model in order to meet the criteria of the Florida Commission on Hurricane Loss Projection Methodology. The importance of sensitivity analysis and uncertainty analysis for a public model whose main purpose is to give appropriate estimates for expected loss costs due to hurricane damages cannot be understated. Sensitivity, as seen in the standardized regression coefficient results, establishes how changes in model input produce changes in model output while uncertainty, as seen in the expected percentage reduction results, studies how much variation in model output is caused by model inputs. The final results presented to the Florida Commission on Hurricane Loss Projection Methodology for sensitivity analysis show that the Holland B parameter is of constant significance, while $\mathrm{R}_{\max }$ increases in significance as the hurricane category increases. This is not only relevant but also consistent with previous models. Furthermore, the relation with the uncertainty, as seen in the expected percentage reduction results, cannot be denied, since the same pattern is exhibited by the Holland $B$ parameter and $R_{\max }$ once more.

The final contour plots produced for the Florida Commission on Hurricane Loss Projection Methodology show an increase in expected loss costs as the hurricane category increases. Within each separate plot a decrease in expected loss costs from east to west can be observed, as is expected due to the weakening of a hurricane on land. Before the final submission for the S-6 form was made, several different contour plots were 
generated using different output results from the model based on changes in the input files. All versions can be found in the USB file containing all documentation.

The creation of a series of $\mathrm{R}$ functions capable of doing the analysis was useful not only in the short term for form S-6, but for any future sensitivity analysis and uncertainty analysis that needs to be done. The code has been thoroughly validated and is properly commented, which makes it possible for anyone with a working knowledge of $\mathrm{R}$ to quickly generate future results provided no major changes occur to the format of the input and output files. Even if such changes were to take place, the core standardized regression coefficients and expected percentage reduction calculations need not be changed, only the sorting of the data into the appropriate format.

Having had the 2005 data for form S-5 as well as the final results in order to both generate the original code and validate it made it possible to create all of the necessary functions months ahead of schedule. Once the first set of output files for form S-6 were released, the necessary changes to the functions were done with plenty of time, so that when the output files generated for form S-6 changed due to changes in the model, regenerating form S-6 results was nearly immediate. This highlights the importance of computer programming for data analysis.

Since the model presented by FIU was approved after very extensive and rigorous review by a panel of experts, the analysis performed can be deemed a success. Future success will be measured based on the usability of the code for future hurricane model submissions. 


\subsection{Recommendations}

The changes from the 2005 to the 2010 output file format made it necessary to both edit the code and revalidate it for any possible inaccuracies. It also made part of the work done when creating the 2005 functions unnecessary and/or invalid. In order to make sure that future submissions to the Florida Commission on Hurricane Loss Projection Methodology of sensitivity analysis and uncertainty analysis are done smoothly using the functions created for form S-6, it is of particular importance that the modeling team maintains consistency in the format of the output files.

The requirements of the Florida Commission on Hurricane Loss Projection Methodology for sensitivity analysis carry the underlying assumption that the hurricane model is linear in nature, since standardized regression coefficients are used. The "SRC gives information on the linear regression model" (Saltelli et al. 1999) but if the model is nonlinear and not monotonic it is necessary to have "SA that is independent from assumptions about the model structure" (Saltelli et al. 1999). Nonlinear model sensitivity analysis methods include the extended FAST and the Sobol. The extended FAST method "is generally more efficient than the method of Sobol" since "the total indices are computationally more expensive than both the correlation/regression measures and screening tests such as that of Morris" (Saltelli et al. 1999). And although "Sobol's method (...) has the capability to calculate higher-order effects, whereas extended FAST is limited to total effects" (Shott) of all factors, the extended FAST method is sufficient for the analysis required by the commission. The extended FAST method computes "the total (all-effects) contribution of each factor to the output variance" (Saltelli et al. 1999) in the manner described by Saltelli in Global Sensitivity Analysis of Model Output. 
It is therefore recommended in the future that sensitivity analysis using the extended FAST method should be conducted. This analysis cannot be done using the current model data, due to the computational cost of the analysis. The current model only has 100 observations available, and for a model with 5 factors the minimum necessary number of model evaluations is 325 . This is given by the formula given by Saltelli et al. (1999):

$$
C=n N_{r} N_{s} \text { where } N_{s}=\left(2 M \omega_{\max }+1\right)
$$

For this cost formula $\mathrm{n}$ is the number of factors, $\mathrm{N}_{\mathrm{r}}$ is the number of curves used (minimum value of 1), and $\mathrm{N}_{\mathrm{s}}$ is defined by $\mathrm{M}$, the interference factor, and $\omega_{\max }$, the largest among the set of frequencies. The sensitivity package created for R (Pujol), which is capable of calculating the extended FAST analysis for a given model has a minimum value for $\mathrm{N}_{\mathrm{s}}$ of 65, which is considered by Saltelli et al (1999) to be a value outside the recommended region due to its small magnitude. 


\section{APPENDICES}

Appendix I: Results for form S-6 presented to FCHLPM

A. Cumulative Relative Frequency Distribution

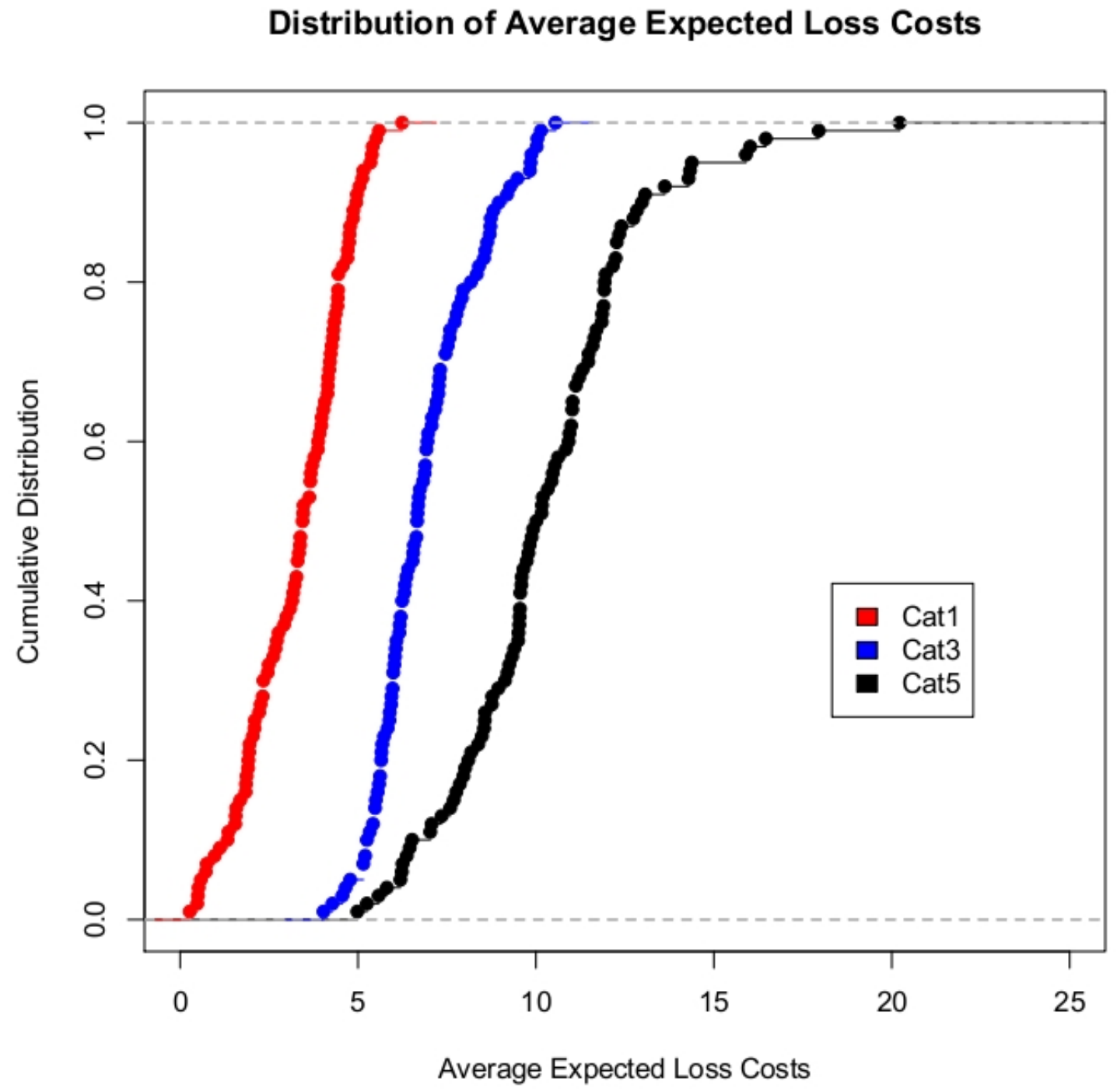


B. Contour Plot for Category 1 Hurricane

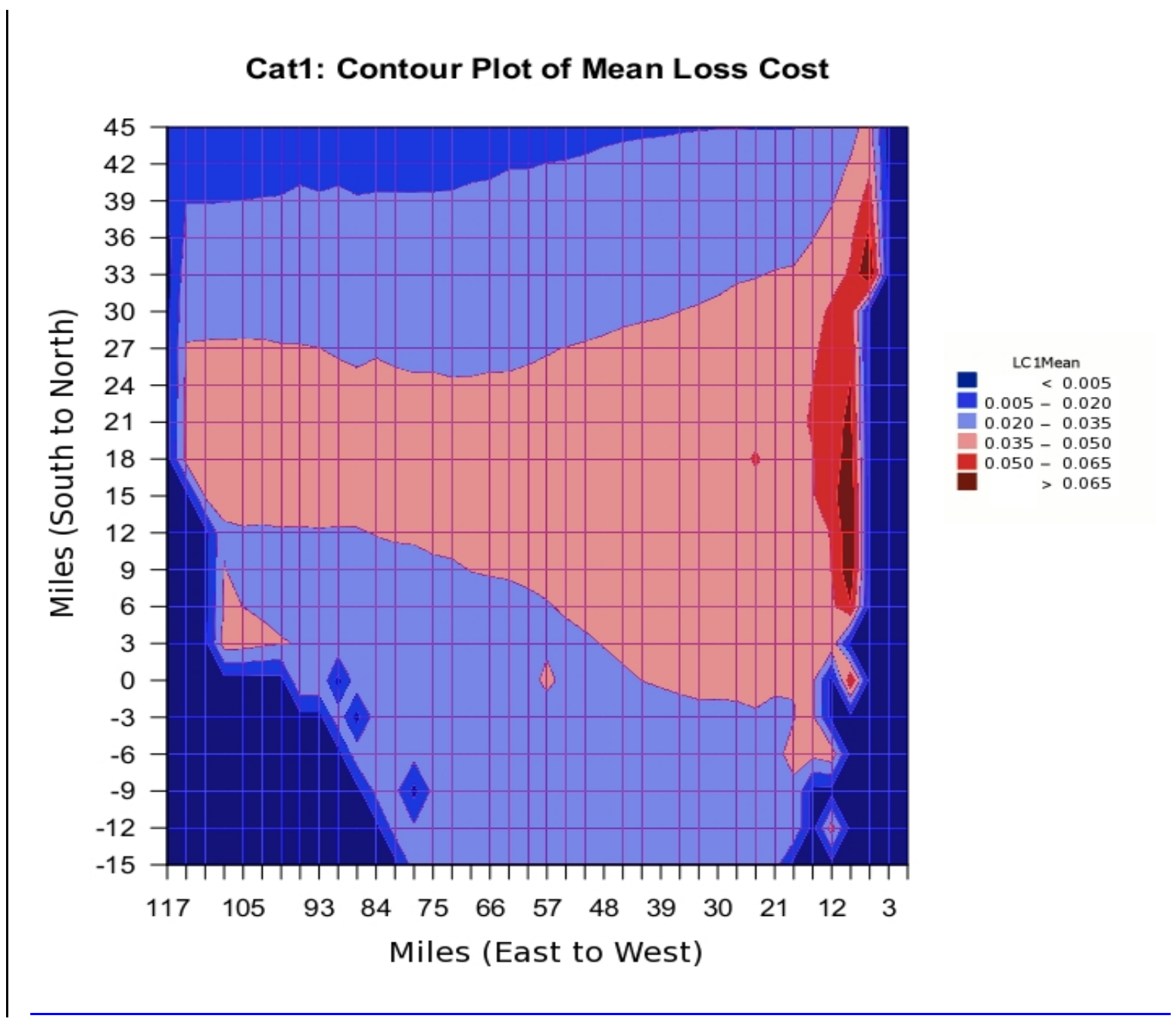


C. Contour Plot for Category 3 Hurricane

Cat3: Contour Plot of Mean Loss Cost
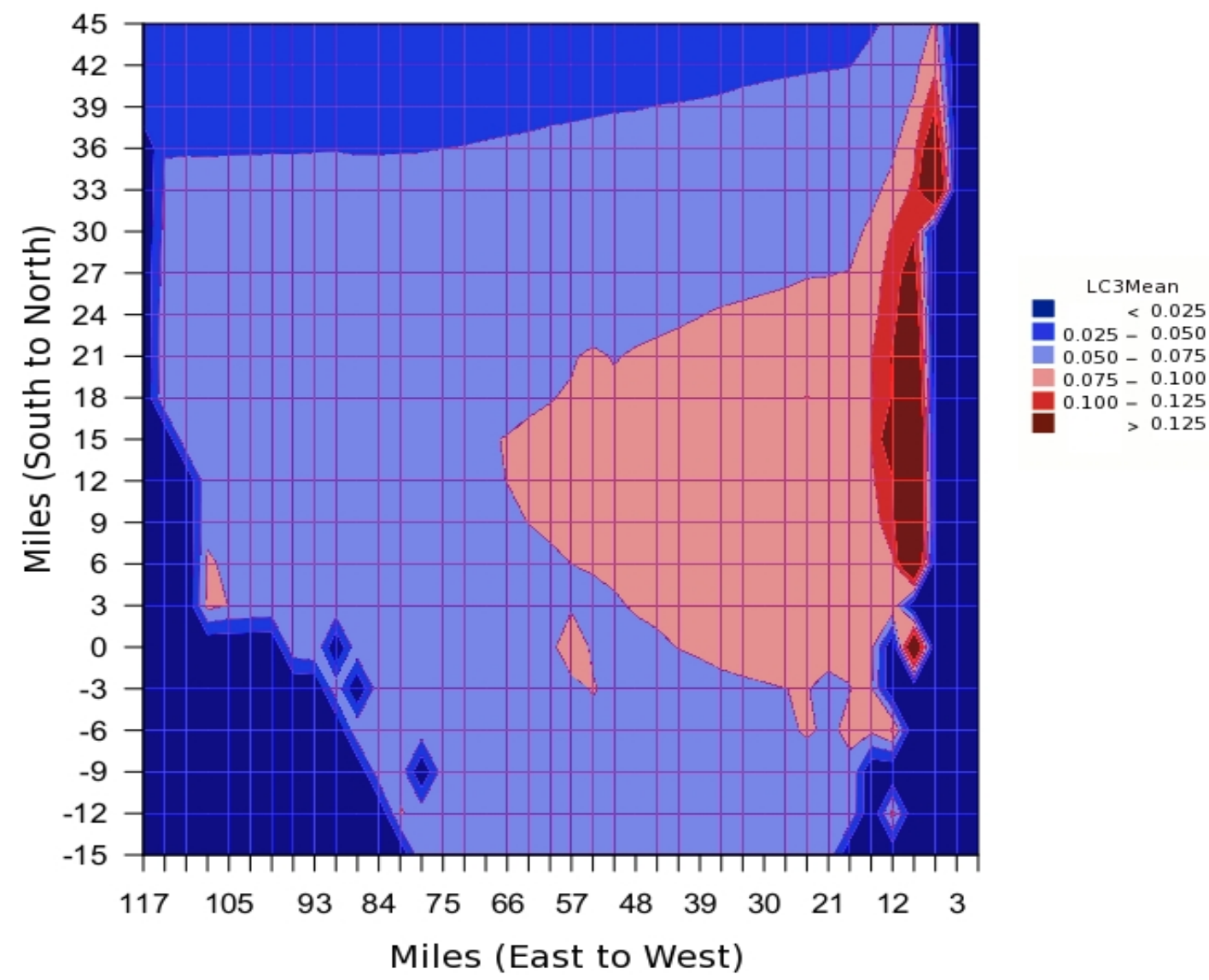
D. Contour Plot for Category 5 Hurricane

Cat5: Contour Plot of Mean Loss Cost

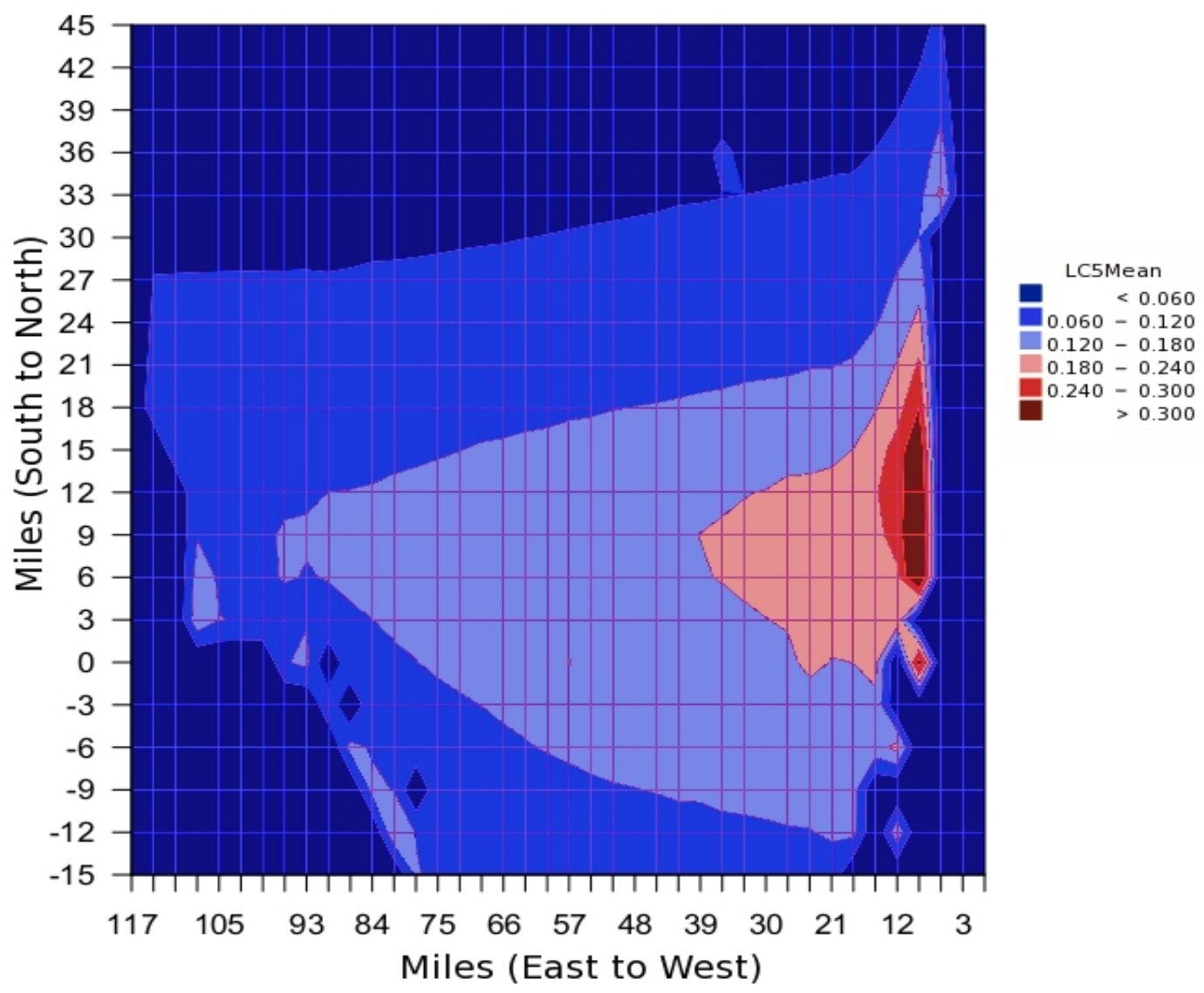




\section{E. Standardized Regression Coefficients by Hurricane Category}

\section{SRC by Hurricane Category}

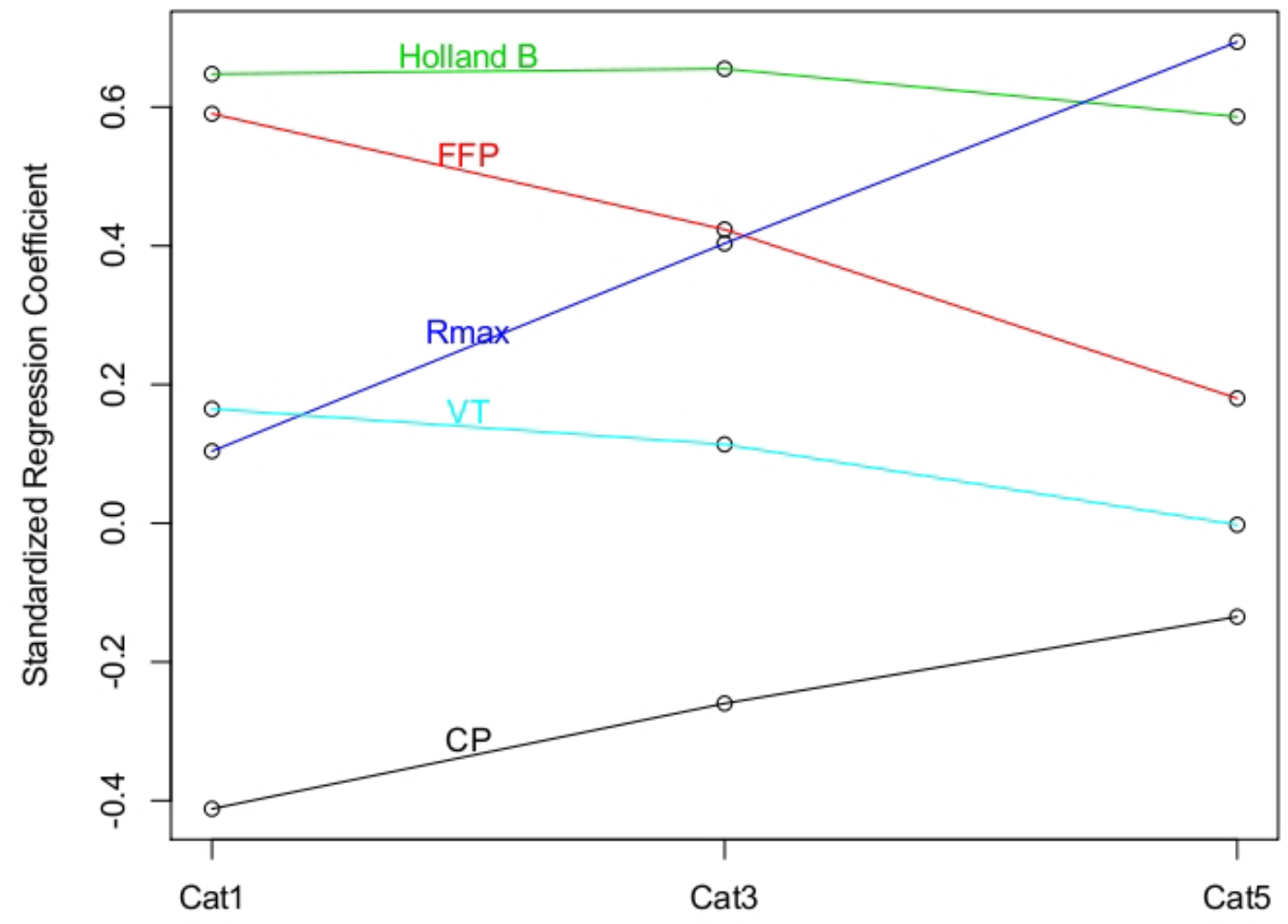

Hurricane Category

\begin{tabular}{|c|c|c|c|c|c|}
\hline Category & $C P$ & $R \max$ & $V T$ & Holland $B$ & $F F P$ \\
\hline 1 & -0.4118 & 0.1039 & 0.1648 & 0.6477 & 0.5905 \\
\hline 3 & -0.2599 & 0.4033 & 0.1137 & 0.6552 & 0.4236 \\
\hline 5 & -0.1349 & 0.6939 & -0.0022 & 0.5862 & 0.1801 \\
\hline
\end{tabular}




\section{F. Expected Percentage Reduction by Hurricane Category}

\section{EPR by Hurricane Category}

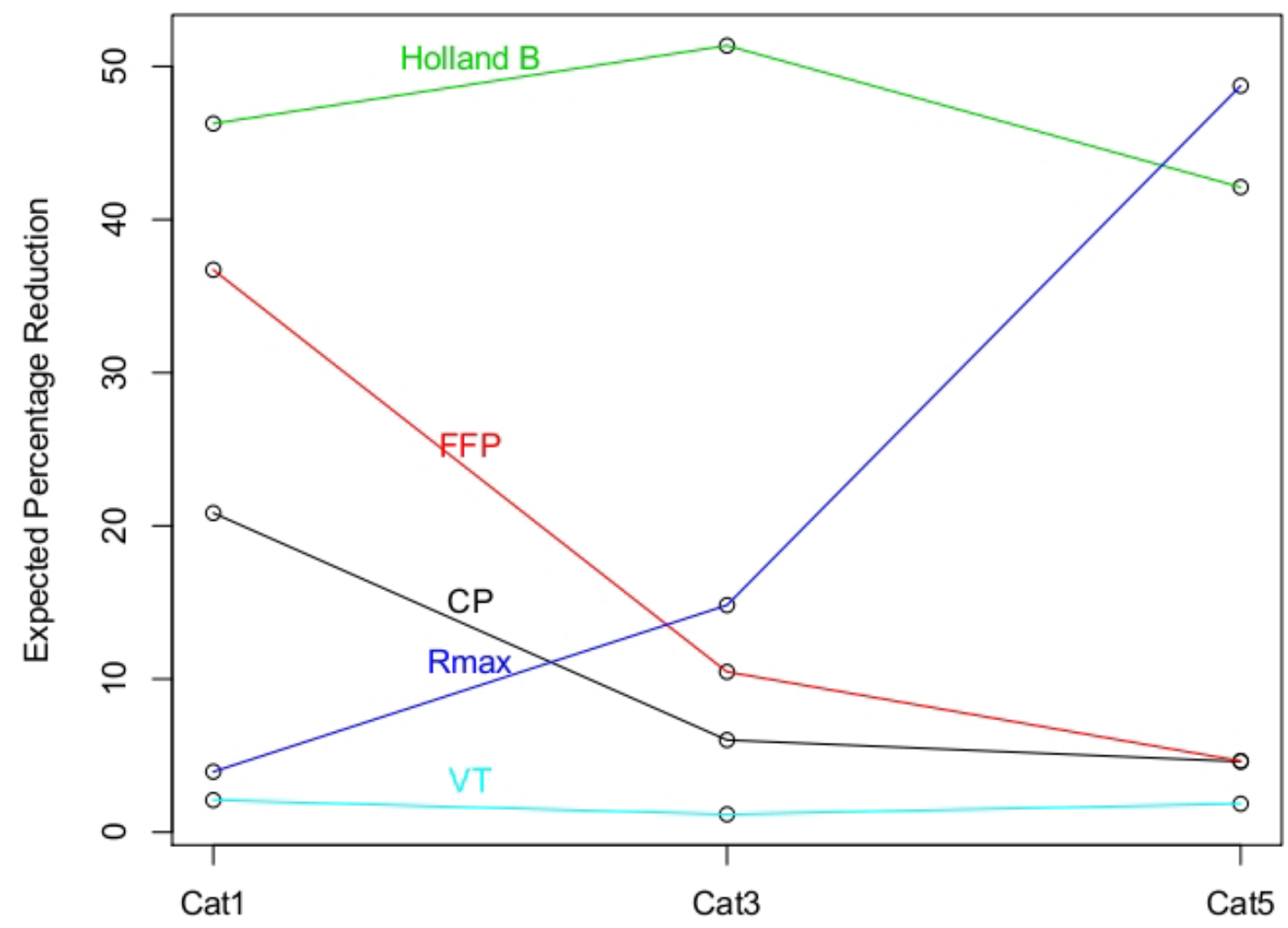

Hurricane Category

\begin{tabular}{|c|c|c|c|c|c|}
\hline Category & $C P$ & Rmax & $V T$ & Holland $B$ & $F F P$ \\
\hline 1 & $20.8398 \%$ & $3.9463 \%$ & $2.0921 \%$ & $46.2717 \%$ & $36.7245 \%$ \\
\hline 3 & $6.0155 \%$ & $14.8201 \%$ & $1.1625 \%$ & $51.3594 \%$ & $10.4668 \%$ \\
\hline 5 & $4.6087 \%$ & $48.7428 \%$ & $1.8529 \%$ & $42.1176 \%$ & $4.6455 \%$ \\
\hline
\end{tabular}




\section{Appendix II: Programming Code for Functions Used}

\section{A. Loading Function}

\#\# Loading Loop loads all appropriate files from working directory and saves into list_of_files the names of all the output files in order, which is used for Uncertainty Calculations.

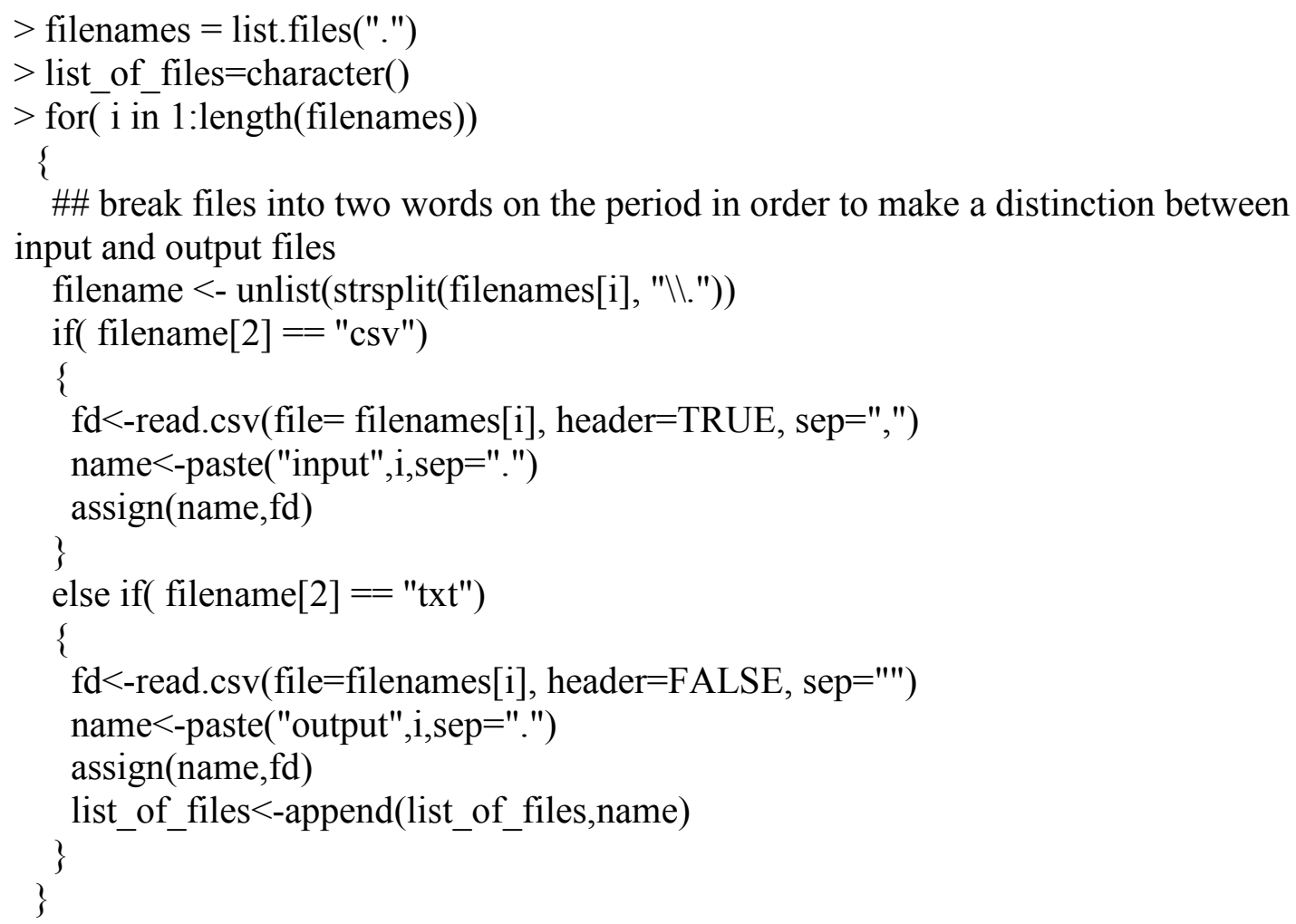

\section{B. SRC Function}

\#\# All code is dependent on the format of the input and output files.

SRCMatrixFunc $<$-function(SA1,SA1Column,inputcat1,inputcat3,inputcat5,HollandB)

\{

\#\# initializes and then creates the three vectors of 100 values for the SA

outputcat $1<$-numeric $(0)$

outputcat $3<$-numeric $(0)$

outputcat $5<$-numeric $(0)$

\#\# Sorts HollandB values to be used throughout the system

HolB $<$-as.matrix(HollandB)

$\mathrm{HolB}<-\mathrm{HolB}[, 16]$

HolB $<$-sort(HolB, decreasing=FALSE) 


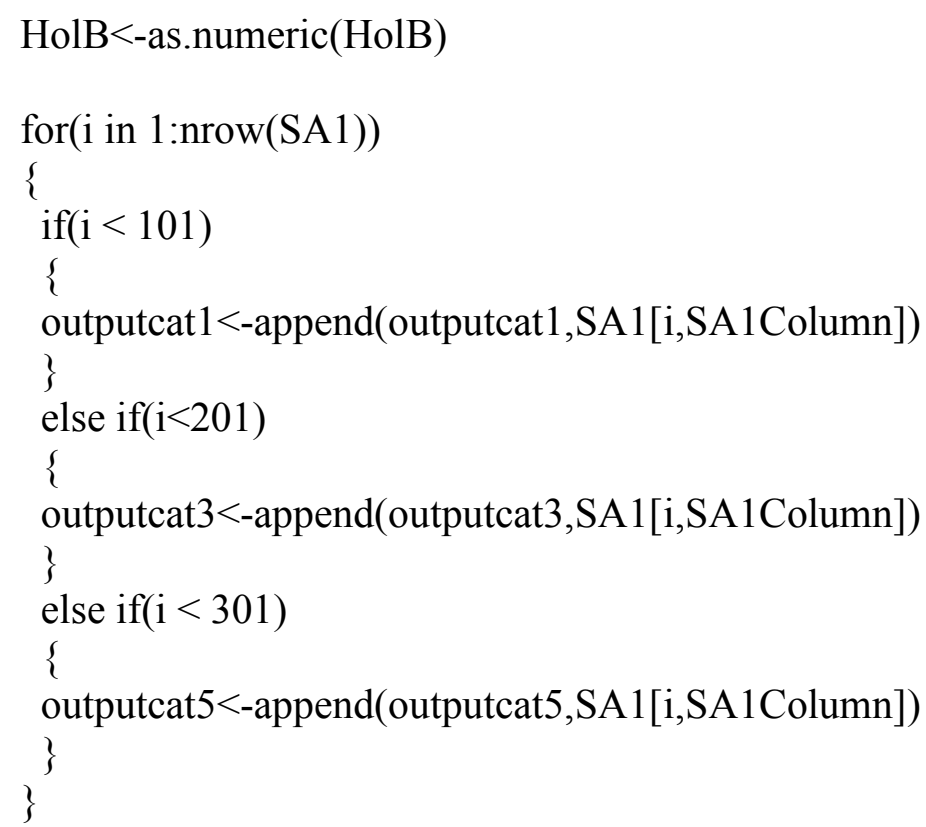

\#\# Creates list of output files, later will use get function to find them to calculate the SRC output_files<-c("outputcat1","outputcat3","outputcat5")

\#\# Creates list of input files, the get function will be used to find them to calculate the SRC input_files<-c("inputcat1","inputcat3","inputcat5")

\#\# Initialize output matrix, the number of rows is 2 less than the number of columns in the input file since both the Quantile parameter and the CF are removed.

SRCMatrix $<$-matrix $($ nrow $=$ ncol(inputcat 1$)-2)$

\#\# Names of rows of final output matrix, SRCMatrix, then removes the name of the 5th column, which is the CF and the 7th, which is the Quantile. It calls it the 6th due to the fact that the removal of the 5 th makes the 7 th the 6 th.

Rows $<$-colnames(inputcat1)

Rows $<-$ Rows[-5]

Rows $<-$ Rows[-6]

\#\# Names of Columns of final output matrix, SRCMatrix

Columns<-c("Cat1", "Cat3", "Cat5")

\#\# loop calculates the SRC for each input/output file combination (there are 3, one for each category hurricane) and appends it to the SRC Matrix

for (i in 1:length(output_files))

\{ 
\#\# maxfinal is a matrix of the original input data, which is called based on the get function in order to append the 100 value vector to it and then calculate the correlation matrix. MeansVector is the vector of 100 values for the output data based on the loop that created the three vectors earlier.

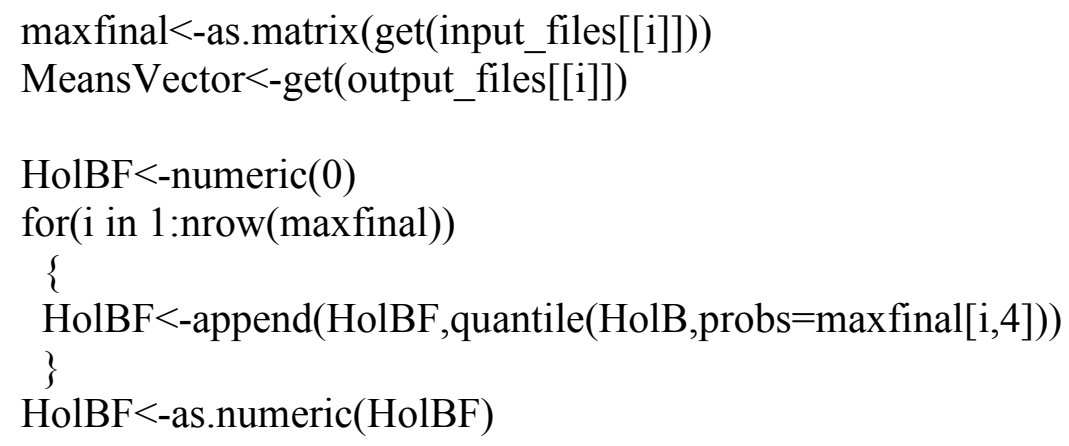

\#\# SensMatrix is the combination of the 100 input values for each one of the variables along with a final column, the MeansVector. Notice that SensMatrix removes the 4th column of maxfinal, which would be the Holland B quantile, and replaces it with the Holland B values.

SensMatrix $<-$ matrix(cbind(maxfinal[,1:3],HolBF,maxfinal[,6],MeansVector),nrow=100)

\#\# Calculates the inverse of the correlation matrix of the SensMatrix, which will be used to calculate the SRC

Cor $1<$-solve (cor(SensMatrix $))$

$\mathrm{SRC}<$-numeric $(0)$

\#\# $\mathrm{q}$ is used to simplify the next loop, which will be the calculation of each SRC. Remember the SRC is the ratio of the last row of the inverse of the correlation matrix to the last element of the inverse of the correlation matrix.

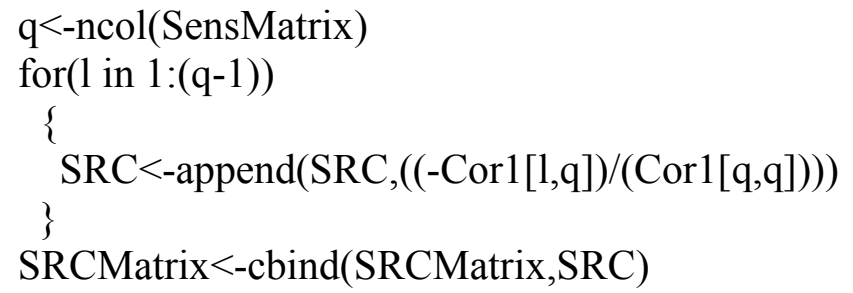

\#\# Removes the initial NA column within SRCMatrix

SRCMatrix<-SRCMatrix[,2:ncol(SRCMatrix)]

\#\# Names both the rows and the columns of the SRCMatrix, then returns the SRCMatrix

SRCMatrix $<-$

matrix(SRCMatrix,ncol=ncol(SRCMatrix),dimnames=list(Rows,Columns)) 


\section{SRCMatrix}

\}

\section{EPR Function}

\#\# Function depends on the format of the output files and their sorted order.

Un

\#\# Initialize vector of variances which will be calculated

VarsVector $<$-numeric $(0)$

MeansVector $<$-matrix(nrow $=100)$

\#\# Loop gets all the .txt files, which are the output files, and for each one it grabs the specified column (VColumn), sorts it appropriately so that MeansVector has Cat1, Cat3, Cat5 for file one, again for file two, etc... and removes the last two files, which are the Holland B track file and the contour plot file.

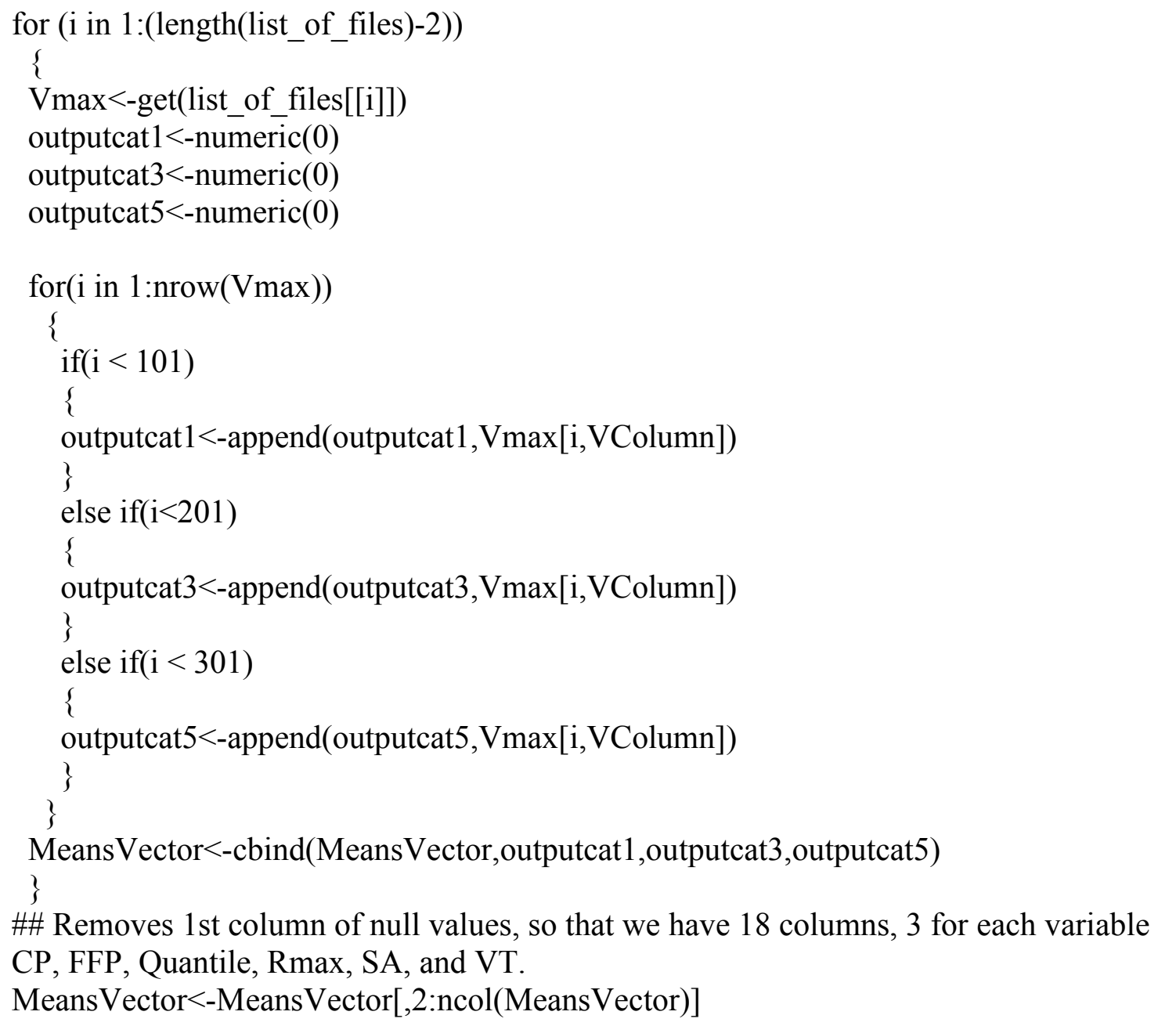


\#\# Calculates the variance for each of the columns and appends it to the variance vector for(j in 1:ncol(MeansVector))

\{

VarsVector<-append(VarsVector,var(MeansVector[,j]))

\}

\#\# VarsMatrix is a matrix which breaks the VarsVector into 3 columns, where each column is the data for one of the hurricanes, First row is CP, second is FFP, 3rd is Quantile, 4th is Rmax, 5th is SA, and 6th is VT.

VarsMatrix $<-$ t(matrix (VarsVector, ncol=6))

UncMatrix $<-$ numeric $(0)$

\#\# Names of rows of UncMatrix

Rows<-c("CP","FFP","Holland B","Rmax","VT")

\#\# Names of Columns of UncMatrix

Columns<-c("Cat1", "Cat3", "Cat5")

\#\# loop calculates the ratio of variances for each of the category hurricanes based on the number of files per category hurricane (including the original output file). The files are ordered such that VarsMatrix[5,] is the original output file.

for (i in 1:length(VarsMatrix))

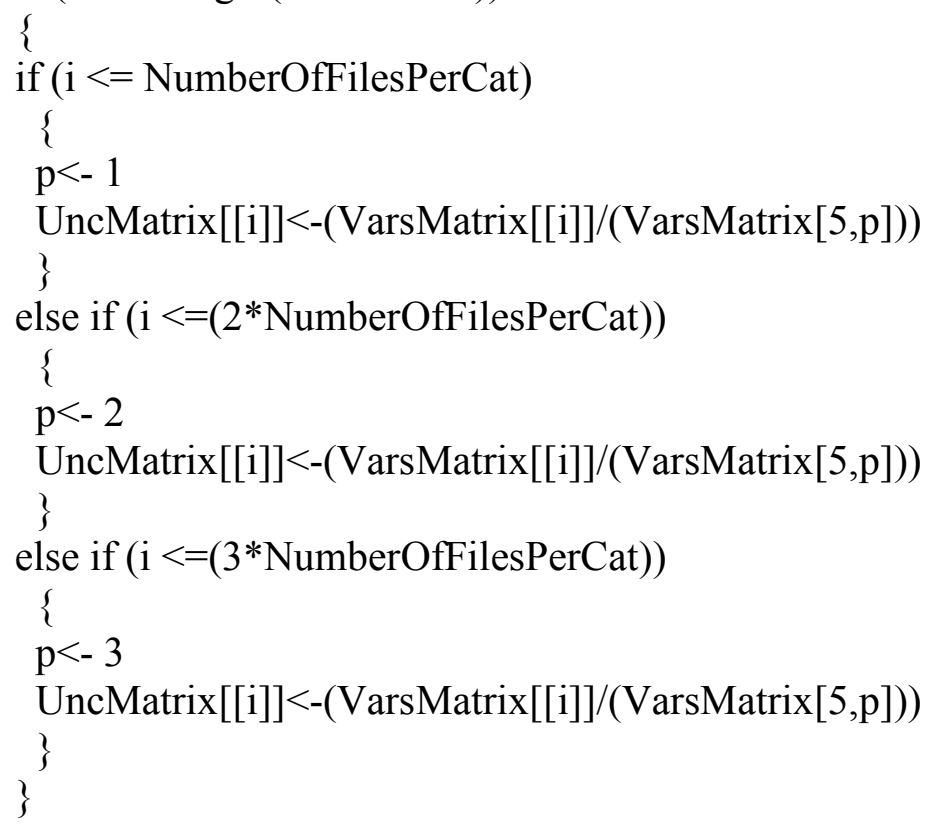

\#\# convert UncMatrix into a matrix with 3 columns, then removes the 5th row, which would be a row of $1 \mathrm{~s}$, and then names the rows and columns of the matrix appropriately. UncMatrix $<$-matrix (UncMatrix,ncol=3)

UncMatrix $<-$ UncMatrix[-5,]

UncMatrix $<-$ UncMatrix*100

UncMatrix<-matrix(UncMatrix,ncol=ncol(UncMatrix), dimnames=list(Rows,Columns)) 


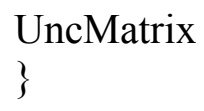

\section{SRC and EPR Plotting Function}

\#\# Simple function can plot both the Sensitivity and Uncertainty matrices.

SUPlotFunction<-function(SUMatrix,Plot.Title,Y.Title)

$$
\begin{aligned}
& \text { \{ } \\
& \text { x.coord }<-c(1,2,3)
\end{aligned}
$$

Plot.Title $<$-as.character(Plot.Title)

Y.Title<-as.character(Y.Title)

plot(col(SUMatrix),SUMatrix,xaxt="n", main=Plot.Title, xlab="Hurricane Category", ylab=Y.Title)

$\operatorname{axis}($ side $=1$, at $=$ x.coord,labels $=$ colnames $($ SUMatrix $))$

for(i in 1:nrow(SUMatrix))

lines(SUMatrix[i,], col=i)

text(1.5,mean(SUMatrix [i,1:2])+max(SUMatrix)/30,labels=rownames(SUMatrix)[i],col=

\section{E. Contour Plot Function}

\#\# Function returns Mean Loss Cost. Must include library(gplots) at the beginning.

$>$ ContourPlot $<-$

function(CatOutput,HurricaneCat,EWEndCoordinate,NSEndCoordinate, Colors,LandWater,NSVertexPoints,Plot.Title)

\{

\#\# Creates matrix of wind values and creates names for the rows and columns of the final matrix based on sequences. EWEndCoordinate and NSEndCoordinate make it possible to adjust the map, since the 2006 and 2010 maps may have different total North-South points $(0,135)$ vs $(0,120)$.

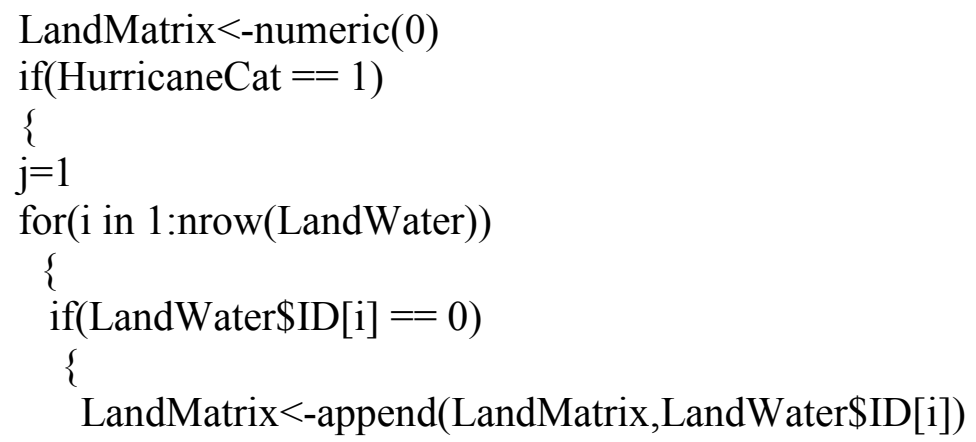




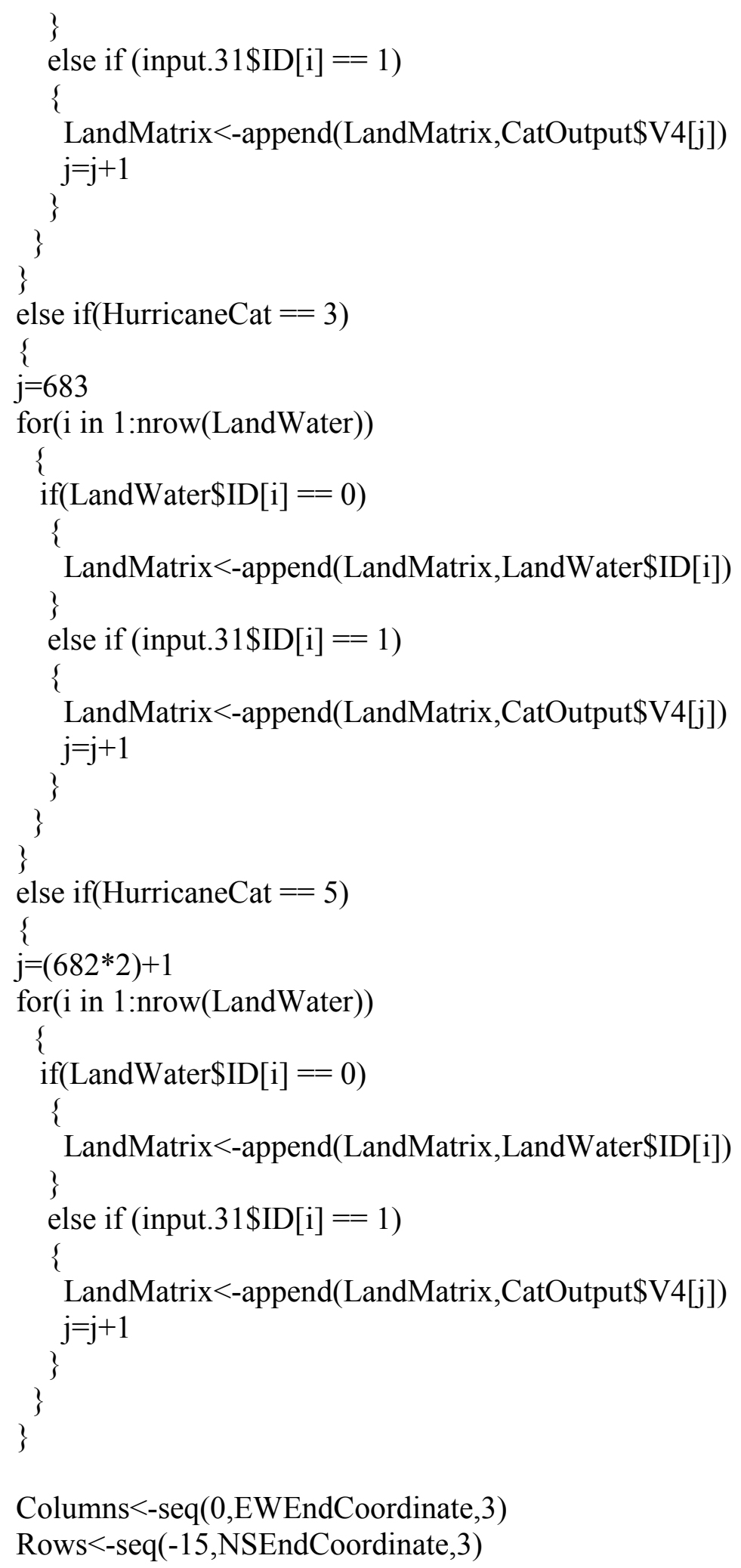

Columns $<$-seq(0,EWEndCoordinate, 3 )

Rows $<$-seq(-15,NSEndCoordinate, 3 ) 
\#\# Makes LandMatrix into matrix with row and column names based on earlier established sequences.

ContourMatrix <-matrix(LandMatrix,nrow=NSVertexPoints, dimnames $=$ list $($ Rows, Columns))

\#\# Rewrites Column Order Backwards to set up for graphing.

ContourMatrix <- ContourMatrix[,ncol(ContourMatrix):1]

\#\# Takes transpose of matrix to prepare it as $\mathrm{x}-\mathrm{y}$ coordinates for graphing

ContourMatrix $<-$ t(ContourMatrix)

\#\# Function within a function, sets up the axes for the graph.

ContourPlotAxes $<-$ function(ContourMatrix)

\{

\#\# Does the y coordinates, from -15 to 45 (columns)

y.coord <- (1:dim(ContourMatrix)[2] - 1) / (dim(ContourMatrix)[2] - 1); y.axis $<-$ axis $($ side $=2$, at $=$ y.coord, labels $=$ colnames $($ ContourMatrix $)$, las $=1)$;

\#\# Does the $\mathrm{x}$ coordinates, from 0 to 135 (rows)

x.coord <- (1:dim(ContourMatrix) [1] - 1) / (dim(ContourMatrix)[1] - 1);

x.axis $<-\operatorname{axis}(\operatorname{side}=1$, at $=x$. coord, labels $=$ rownames(ContourMatrix $)$, las $=1)$;

\}

\#\# Creates the plot to be seen, with variable colorpanel number and title. Levels were edited within the code for each category hurricane due to last minute changes required in the plotting.

Plot.Title $<$-as.character(Plot.Title)

ContourCat<-filled.contour(ContourMatrix, col=colorpanel(Colors, "blue","red"), levels $=\mathrm{c}(.00, .005, .010, .015, .020, .025, .1)$, plot.axes $=$ ContourPlotAxes(ContourMatrix), main=Plot.Title)

ContourCat \}

\section{F. CRF Function}

plot(ecdf(output.29[201:300,4]),xlim=c(0,25),main="Distribution of Average Expected Loss Costs",xlab="Average Expected Loss Costs",ylab="Cummulative Distribution") lines(ecdf(output.29[101:200,4]),col='blue')

lines(ecdf(output.29[1:100,4]),col='red')

legend(locator(n=1),c("Cat1","Cat3","Cat5"),fill=c("red","blue","black")) 


\section{References}

1. Chambers, J. M. (2008). Software for Data Analysis: Programming with R. New York, NY: Springer Science + Business Media.

2. Florida Commission on Hurricane Loss Projection Methodology, Report of Activities as of November 1, 2007.

3. Hamid, S., Kibria, B.M.G, Gulati, S. et.al (2008). Public hurricane loss evaluation models: predicting losses of residential structures in the State of Florida. JSM 2008 proceedings, 1052-1059.

4. Hamid, S., Kibria, B.M.G., Gulati, S. et al. (2010). Predicting losses of residential structures in the State of Florida by the public hurricane loss evaluation models with discussion. Statistical Methodology, $\underline{7}, 552-573$.

5. Iman, R. L., Johnson, M. E., and Schroeder, T. (2002a). Assessing Hurricane Effects. Part 1. Sensitivity Analysis, Reliability Engineering \& System Safety, $\underline{78}$, 131-145.

6. Iman, R. L., Johnson, M. E., and Schroeder, T. (2002b). Assessing Hurricane Effects. Part 2. Uncertainty Analysis, Reliability Engineering \& System Safety, $\underline{78}, 147-155$.

7. Los Alamos Scientific Laboratory (1979). Sensitivity Analysis. United States Department of Energy, Los Alamos, New Mexico: U.S. Government Printing Office.

8. Pujol, G. (2012). Sensitivity Analysis. Unpublished Manuscript. Retrieved from: http://cran.r-project.org/web/packages/sensitivity/sensitivity.pdf

9. Saltelli, A., Tarantola, S., Chan, K. P.-S. (1999). A Quantitative ModelIndependent Method for Sensitivity Analysis of Model Output. Technometrics, $\underline{41}, 39-56$.

10. Shott, G. J., Yucel, V., Desotell, L., Pyles, G., Carilli, J. (2007). “Uncertainty and Sensitivity of Alternative Rn-222 Flux Density Models Used in Performance Assessment." Waste Management Symposia. February 21-March 1, 2007, Tucson, AZ.

11. Spector, P. (2008). Data Manipulation with R. New York, NY: Springer Science + Business Media. 
12. The R Project. (n.d.) The R Project for Statistical Computing. Retrieved from the R project website: http://www.r-project.org/index.html

13. Tran, E. (1999). Verification/Validation/Certification. Carnegie Mellon University. Unpublished Manuscript. Retrieved from:

http://www.ece.cmu.edu/ koopman/des_s99/verification/index.html\#verification techniques.

14. Vickery, P. J., Whadhera, D. (2008). " Statistical Models of Holland Pressure Profile Parameter and Radius to Maximum Winds of Hurricanes from FlightLevel Pressure and H*Wind Data." Journal of Applied Meteorology and Climatology. 47, 2497-2517. 